\title{
Article
}

\section{Parametric Predictions for Pure Electric Vehicles}

\author{
Bukola Peter Adedeji (D)
}

\section{check for}

updates

Citation: Adedeji, B.P. Parametric Predictions for Pure Electric Vehicles. World Electr. Veh. J. 2021, 12, 257. https://doi.org/10.3390/ wevj12040257

Academic Editor: Joeri Van Mierlo

Received: 14 September 2021 Accepted: 30 November 2021 Published: 8 December 2021

Publisher's Note: MDPI stays neutral with regard to jurisdictional claims in published maps and institutional affiliations.

Copyright: (c) 2021 by the authors. Licensee MDPI, Basel, Switzerland. This article is an open access article distributed under the terms and conditions of the Creative Commons Attribution (CC BY) license (https:// creativecommons.org/licenses/by/ $4.0 /)$.
Department of Industrial Systems Engineering, University of Regina, Regina, SK S4S 0A2, Canada; adedejib@uregina.ca

\begin{abstract}
Demand for pure electric vehicles has been found to be increasing over the years. This has necessitated the development of a model that would serve as a predicting machine for manufacturing different types of pure electric vehicles. Direct Artificial Neural Network approach was used for predictions of nine different parameters commonly found in pure electric cars. Predictions were found to be of high degree of accuracy while using unit and overall model errors as the basis of performance measurement. The mean absolute error, mean square error and root mean square error of the model were $0.109,0.218$ and 0.467 , respectively, when the combined electric charge consumption was used for modeling. For the model formation, using the same variable, the losses for the training and testing were $3.9132 \times 10^{-6}$ and $9.698 \times 10^{-7}$, respectively. The model was also evaluated using redefined datasets. The developed model can be used by manufacturers and engineers to simulate future designs when certain parameters are given.
\end{abstract}

Keywords: pure electric vehicles; prediction; artificial neural networks; model; variables; datasets

\section{Introduction}

The world is moving rapidly to combat the emission of greenhouse gases. Of course, emissions from the vehicles are important factors that are destroying our planet. To curb this, world leaders are massively investing in pure electric vehicles (PEVs) and hybrid electric vehicles (HEVs). For instance, President Biden of the United of America, in 2021 after his inauguration proposed an investment plan for installation of charging stations and supports for PEVs manufacturers. He also proposed that all vehicles in the United States by 2040 would be electric [1]. Sales and demands of PEVs are increasing drastically on yearly basis in Canada according to data [2]. To facilitate the production of PEVs, it is expedient to develop a toolbox for predicting their parameters. This will help the manufacturing engineers to be able to simulate future designs more accurately. This study presents the toolbox or the simulating technique for facilitating the design of PEVs.

Safak [3] classified electrified vehicles into four categories, namely: battery electric vehicles (BEVs), fuel-cell-electric-vehicles (FCEVs), plug-in hybrid electric vehicles (PHEVs), and hybrid electric vehicles (HEVs). The findings in this study will focus on BEVs or PEVs. It should be noted that pure electric vehicles are also referred to as all-electric vehicles (AEVs) or battery electric vehicles (BEVs) in the literatures [3,4]. PEVs do not require internal combustion engines for their propulsion. They are powered by an electric motor only. Modern PEVs manufactured in year 2021 have longer ranges. Examples are Tesla Model X and Tesla Model Y, with ranges $580 \mathrm{~km}$ and $358 \mathrm{~km}$, respectively [2]. Another example is Lucid car which has a range of $520 \mathrm{~m}$, that is about $836 \mathrm{~km}$ [5].

Direct models of Artificial Neural Network (ANN) or neural network approaches have been applied to different categories of electrified vehicles in the literatures [6-9]. For example, ANN methods have been applied to battery technologies [10-13] and energy management [14-19]. Wang et al. [14] developed a multi-neural network controller based on Kernel Fuzzy C-means Clustering (KFCM) for energy management of hybrid electric vehicles. The controller was embedded in an ADVISOR controller for the purpose of forward simulation process. Shi et al. [15] equally used KFCM for multi-neural network 
(MNN) to improve the fuel economy of a parallel hybrid electric vehicle. MNN was used as the output of the energy management system. The result of the work indicated that 'KFCM-MNN has good learning simulation' capability. It also shows that KFCM-MNN had better performance in terms of improvement of fuel economy than a single neural network controller.

Zhu et al. [20] applied Deep Learning to forecast or predict super-short-term stochastic charging load in plug-in electric vehicles. The research was conducted using 12 vehicles. The finding shows that deep learning has high accuracy for forecasting super-short-term charging load for plug-in electric vehicles.

A study was conducted using structural equation model (SEM) and artificial neural networks to identify and rank five factors that could affect the decision of customers to purchase pure electric vehicles (PEVs). The result of the study can help the policy makers to implement things that will encourage customers to purchase PEVs [21].

Huang et al. [22] employed deep artificial neural network to evaluate the quality of sound in pure electric vehicles. The result of the prediction shows better accuracy when compared to conventional backpropagation neural network. Qian et al. [23] used geneticoptimized backpropagation artificial neural network (GA-PB ANN) to estimate the sound quality of pure electric vehicles. The finding of the research shows that GA-PB ANN has better precision than multiple linear regression model.

This paper proposes a direct model of Artificial Neural Networks application to manufacturing of pure electric vehicles. The objective of the study is to develop a model that can predict nine indispensable design-parameters of PEVs. The study will aid manufacturing of PEVs in data-driven and nonlinear manners. The developed model would assist in decision making in terms of parameter selection. This study uses datasets of $223 \mathrm{PEVs}$. The categories of vehicles used include two-seater, full-size, compact, subcompact, mid-size, standard SUV, and small station wagons. Examples of make of cars used are Mitsubishi, Nissan, Ford, Smart, Tesla, Chevrolet, BMW, Kia, Hyundai, Audi, Volkswagen, Porsche, and Volvo.

\section{Materials and Methods}

ANNs are used for solving nonlinear problems. They are also suitable for predictions with high degree of accuracy. The most common application of an ANN is a direct model approach. An inverse model approach is more tedious and complicated. The proposed method implemented in this study is known as Multi Layer Perceptron (MLP); and can be considered as a Direct model of Artificial Neural Network (DANN or ANN ${ }_{D}$ ). The MLP used in this study employs feedforward backpropagation algorithms. During the feedforward process, errors are determined while moving from the input side to the output end. For the backpropagation process, which occurs after the feedforward process, errors are minimized by updating the weights from the output side to the input end. This is carried out layer by layer. The minimization of the error is done by using an optimizer.

\subsection{Dynamic Control Process}

One of the attributes of ANN is that it can be used to model and control a dynamic system [24]. In a direct ANN dynamic control process, raw datasets of input variables are used with those of the target variable to predict the outputs. The process of prediction can be subdivided into three stages. The stages are the training, testing/validation, and actual simulation. However, it should be noted that ANN consists of nodes (neurons), layers (such as input, hidden and output), and the connecting weights [24-27]. The dynamic control process is represented by Figure 1. During the dynamic control process, errors functions may be applied to determine the losses. There are different types of error functions. The suitable ones for this case under consideration were applied. The values of the errors are also minimized by updating the weights. 


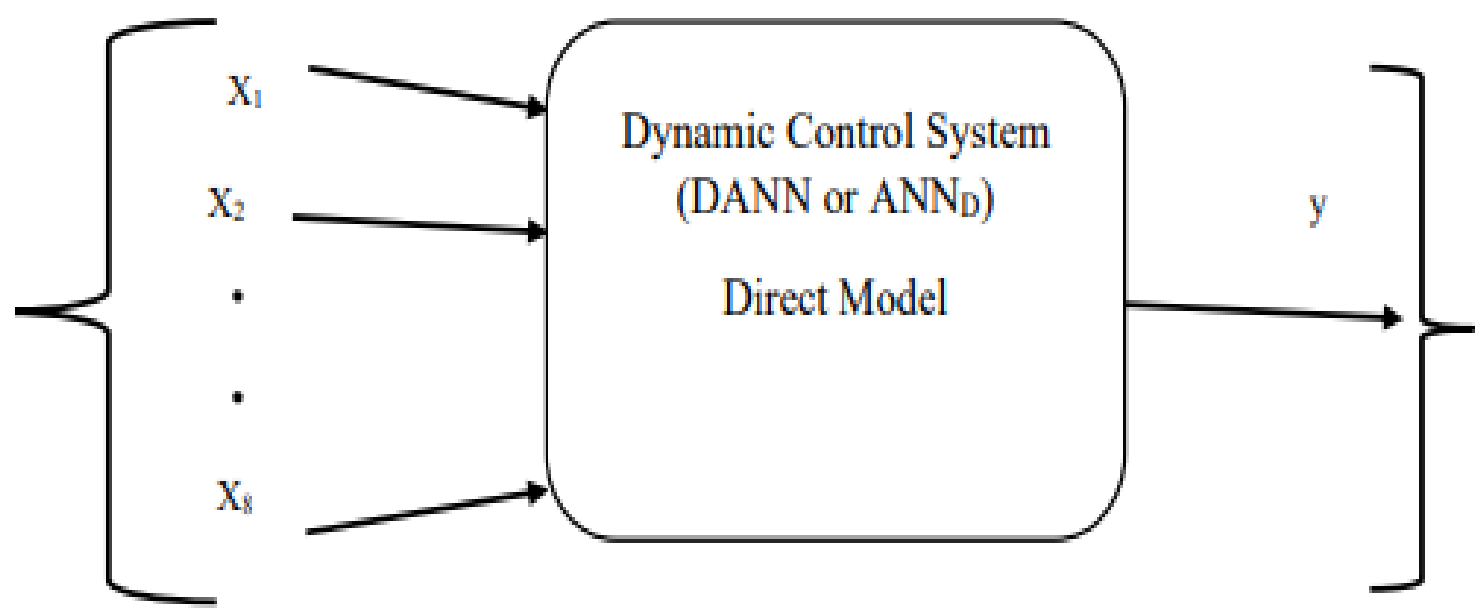

Figure 1. Dynamic Control System for the PEVs.

\subsubsection{The Training Process}

There are nine trainable variables in the datasets of the electric vehicles as show in Table 1. Out of the nine variables, eight were used as the inputs and one was used as the target output. Hence, this can be called a single-output system. Figure 1 shows the dynamic control system. For the training of the data, the following sets are needed:

$$
\left\{x_{1}, x_{2}, \ldots, x_{n}\right\}_{k} \quad \text { for the input variables }
$$

$\left\{\mathrm{y}_{1}, \mathrm{y}_{2}, \ldots, \mathrm{y}_{\mathrm{m}}\right\}_{\mathrm{k}} \quad$ for the target output variables

$$
\begin{aligned}
& X=\left\{x_{1}, x_{2}, \ldots, x_{n}\right\}_{k} \in R^{n} \\
& Y=\left\{y_{1}, y_{2}, \ldots, y_{m}\right\}_{k} \in R^{n}
\end{aligned}
$$

where $\mathrm{k}=$ number of the input and output pairs that are fed into the system in Figure 1.

Table 1. Datasets for the model.

\begin{tabular}{cccccccccccc}
\hline & Year & Make & Mot & CE & HE & ComE & CGEq & HGEq & ComGEq & Ra & ReT \\
\hline 0 & 2012 & Mitsubishi & 49 & 16.9 & 21.4 & 18.7 & 1.9 & 2.4 & 2.1 & 100 & 7 \\
1 & 2012 & Nissan & 80 & 19.3 & 23 & 21.1 & 2.2 & 2.6 & 2.4 & 117 & 7 \\
2 & 2013 & Ford & 107 & 19 & 21 & 20 & 2.1 & 2.4 & 2.2 & 122 & 4 \\
3 & 2013 & Mitsubishi & 49 & 16.9 & 21.4 & 18.7 & 1.9 & 2.4 & 2.1 & 100 & 7 \\
4 & 2013 & Nissan & 80 & 19.3 & 23 & 21.1 & 2.2 & 2.6 & 2.2 & 117 & 7 \\
5 & 2013 & Smart & 35 & 17.2 & 22.5 & 19.6 & 1.9 & 2.5 & 2.2 & 109 & 8 \\
6 & 2013 & Smart & 35 & 17.2 & 22.5 & 19.6 & 1.9 & 2.5 & 2.2 & 109 \\
7 & 2013 & Tesla & 225 & 22.4 & 21.9 & 22.2 & 2.5 & 2.5 & 2.5 & 224 & 6 \\
8 & 2013 & Tesla & 225 & 22.2 & 21.7 & 21.9 & 2.5 & 2.4 & 2.5 & 335 \\
9 & 2013 & Tesla & 270 & 23.8 & 23.2 & 23.6 & 2.7 & 2.6 & 2.6 & 426 \\
10 & 2013 & Tesla & 310 & 23.9 & 23.2 & 23.6 & 2.7 & 2.6 & 2.6 & 426 \\
11 & 2014 & Chevrolet & 104 & 16 & 19.6 & 17.8 & 1.8 & 2.2 & 2 & 12 \\
12 & 2014 & Ford & 107 & 19 & 21.1 & 20 & 2.1 & 2.4 & 2.2 & 131 \\
13 & 2014 & Mitsubishi & 49 & 16.9 & 21.4 & 18.7 & 1.9 & 2.4 & 2.1 & 7 \\
14 & 2014 & Nissan & 80 & 16.5 & 20.8 & 18.4 & 1.9 & 2.3 & 2.1 & 100 \\
\hline
\end{tabular}

where $\mathrm{R}$ is a real number.

$$
\mathrm{Y}=\mathrm{f}(\mathrm{x})
$$

For the case under consideration, we do not know $f(x)$, but we have sufficient data pairs for inputs and outputs. Therefore, 


$$
\{\mathrm{x}\}_{\mathrm{k}} \leftrightarrow\{\mathrm{y}\}_{\mathrm{k}}
$$

The following steps are required for the training:

- The input variables are randomly selected from the database in such that,

$$
\begin{aligned}
& \{X\}_{k}=\left\{x_{1}, x_{2}, \ldots, x_{n}\right\}_{k} \\
& k=1,2,3, \ldots, k_{2} \leq k_{1}
\end{aligned}
$$

In practice, $\mathrm{k}_{1} \approx 500$ iterations when $\mathrm{f}(\mathrm{x})$ is not known.

The output variable are also randomly picked such that,

$$
\{Y\}_{k}=\left\{y_{1}, y_{2}, \ldots, y_{m}\right\}_{k}
$$

- $\{X\}_{\mathrm{k}}$ is fed as the input into the neural network.

- $\quad\{\mathrm{Y}\}_{\mathrm{k}}$ is fed as the target output into the neural network.

- The feeding is carried out $\mathrm{k}_{2}$ times.

- The training of the network is carried out until the overall error " $E$ " is a small value.

- If the value of the overall error $E$ is large, then, another randomly pair of input and output are selected. The above process would be repeated.

\subsubsection{Testing/Validation Process}

After the ANN has beeen satisfactorily trained, that is when all the error criterial have been satisfied, the following steps are to be employed:

- A new set of input datasets $\{X\}_{\mathrm{k}}$ is selected and the corresponding outputs of these input variables are recorded as $\{\mathrm{Y}\}_{\mathrm{k}}$.

- This new set $\{X\}_{k}$ is fed into the ANN as the agent of testing for $k=1,2,3, \ldots, k_{3}$ and the corresponding value of the actual output $\overline{\{Y}_{\mathrm{k}}$ is recorded.

- The value of the error is calculated using the recorded outputs $\{Y\}_{k}$ and $\left\{\overline{Y Y}_{k}\right.$ above.

$$
\begin{gathered}
\varepsilon=\|\mathrm{Y}-\overline{\mathrm{Y}}\|^{2} \\
E=\frac{1}{2} \sum_{\mathrm{k}=1}^{\mathrm{k}_{3}} \varepsilon
\end{gathered}
$$

Substituting for the value of local error $\varepsilon$, the total error becomes,

$$
E=\frac{1}{2} \sum_{\mathrm{k}=1}^{\mathrm{k}_{3}}\|\mathrm{Y}-\overline{\mathrm{Y}}\|^{2}
$$

The actual output variable is $\mathrm{y}_{1}$. The input variables are eight in number $\left\{\mathrm{x}_{1}, \mathrm{x}_{2}, \ldots, \mathrm{x}_{8}\right\}$.

$$
\begin{gathered}
\{Y\}_{k}=\left\{y_{1}\right\}_{k} \\
\{X\}_{k}=\left\{x_{1}, x_{2}, \ldots, x_{8}\right\}_{k}
\end{gathered}
$$

\subsection{DANN Process Flow for Parametric Predictions}

The process flow for the prediction in this study can be subdivided into six major steps. These steps are discussed in detail below. Figure 2 shows the details of the process flow. 


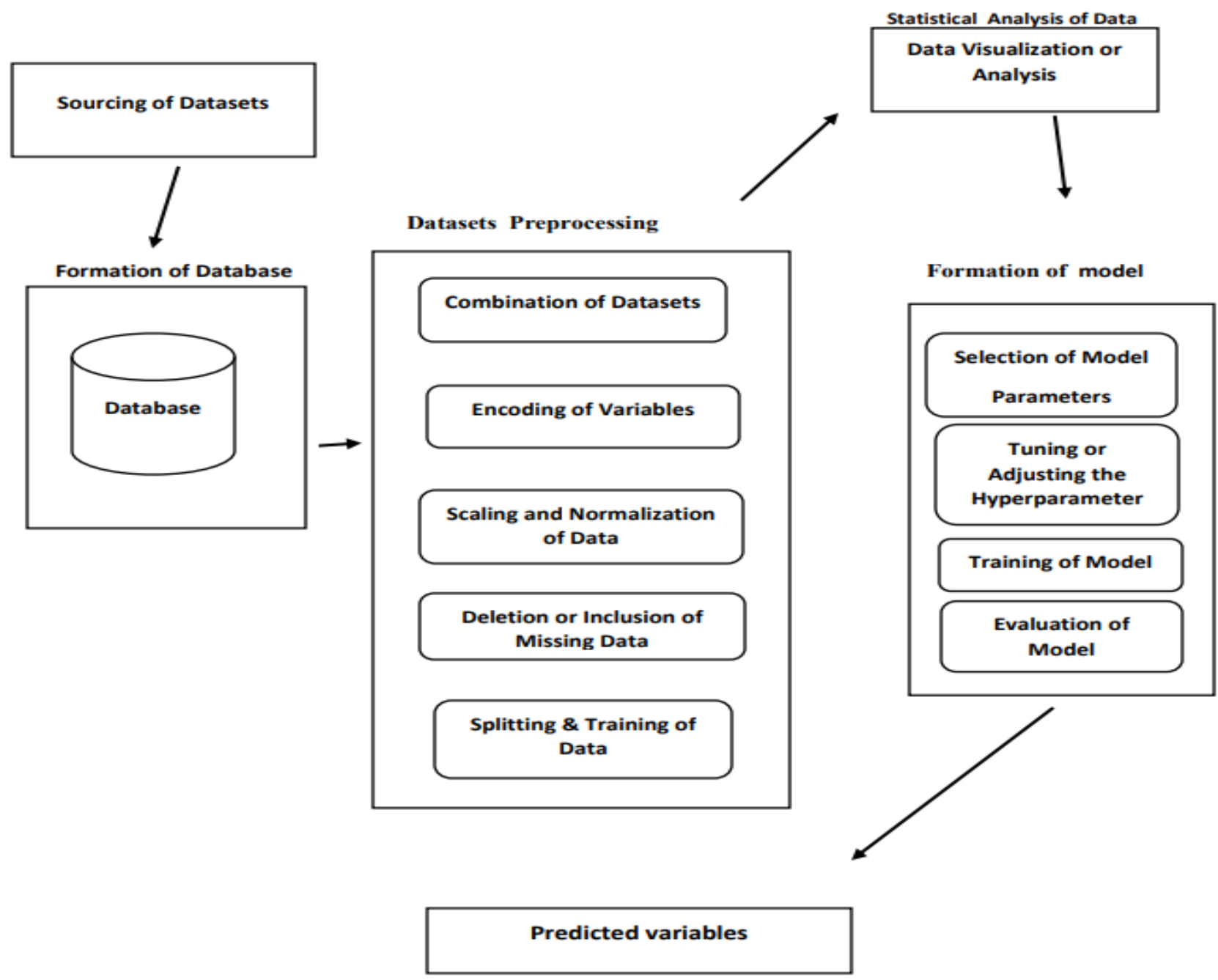

Figure 2. Process flow for the parametric predictions.

\subsubsection{Data Sourcing}

Datasets were collected from the Ministry of Transportation Canada [2]. The original datasets were experimental or raw data from the automobile manufacturers.

\subsubsection{Database Formation}

Database of pure electric vehicles with various parameters was created using Python library called Panda as shown in Table 1. The datasets consist of various models or makes of PEVs or battery electric vehicles.

\subsubsection{Data Preprocessing}

Datasets from different manufacturers of PEVs were processed in a tabular form. Parameters that were trainable were selected and the remaining were eliminated. The needed datasets were assigned specific names for the purpose of identification as shown in Table 1. Datasets were normalized and scaled using standard scaler to reduce the wide merging between the datasets. Big datasets were squash (or compress) by this process. Datasets were divided into training and testing/validation data at ratio 0.8 and 0.2 , respectively.

To code in Python libraries, the following variables listed below were used. 


\section{Variables}

$\mathrm{x}_{1}=$ Mot-Electric motor power in $\mathrm{kW}$

$\mathrm{x}_{2}=\mathrm{CE}-$ City electric charge consumption $(\mathrm{kWh} / 100 \mathrm{~km})$

$\mathrm{x}_{3}=\mathrm{HE}$-Highway electric charge consumption $(\mathrm{kWh} / 100 \mathrm{~km})$

$\mathrm{y}_{1}=$ ComE-Combined electric charge consumption $(\mathrm{kWh} / 100 \mathrm{~km})$

$\mathrm{x}_{4}=\mathrm{CGEq}-$ City gasoline litre consumption equivalent $(\mathrm{Le} / 100 \mathrm{~km})$

$\mathrm{x}_{5}=\mathrm{HGEq}-$ Highway gasoline litre consumption equivalent $(\mathrm{Le} / 100 \mathrm{~km})$

$\mathrm{x}_{6}=$ ComGEq-Combined highway and city gasoline litre consumption equivalent $(\mathrm{Le} / 100 \mathrm{~km})$

$\mathrm{x}_{7}=\mathrm{Ra}-$ Range (the estimated driving distance (in kilometers) on a fully charged battery)

$\mathrm{x}_{8}=\mathrm{ReT}$-Recharge time in hours

where Le is the gasoline litre equivalent and $\mathrm{kWh}$ is the kilowatt hour of electricity.

\subsubsection{Statistical Analysis of Data}

The selected datasets were analyzed by using statistical parameter to see how they were distributed. This is known as data visualization. Histogram was used to visualize each of the parameters as shown in Figure 3.

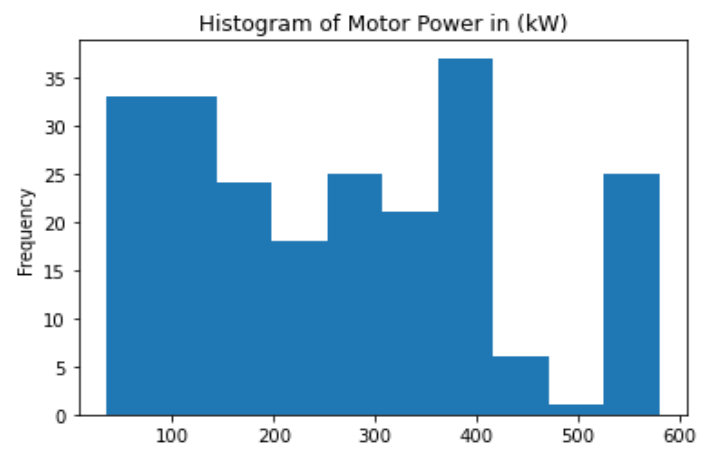

(a)

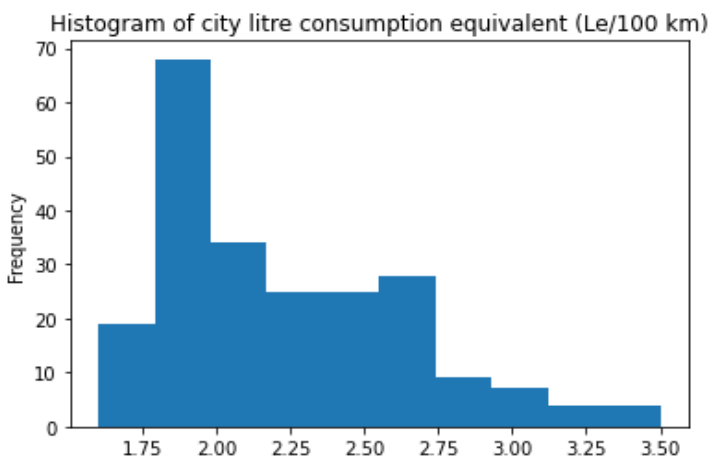

(c)

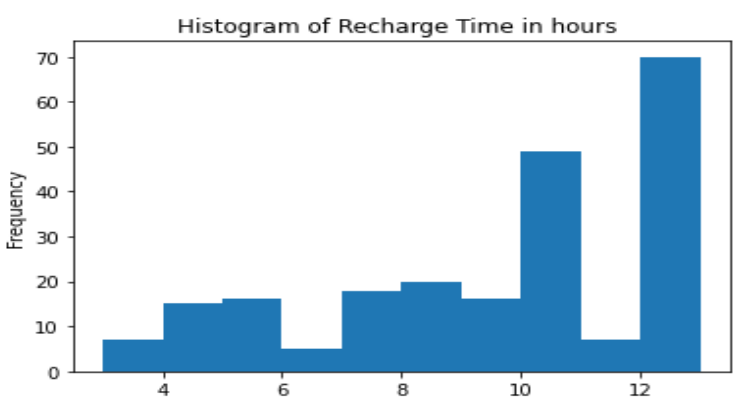

(e)

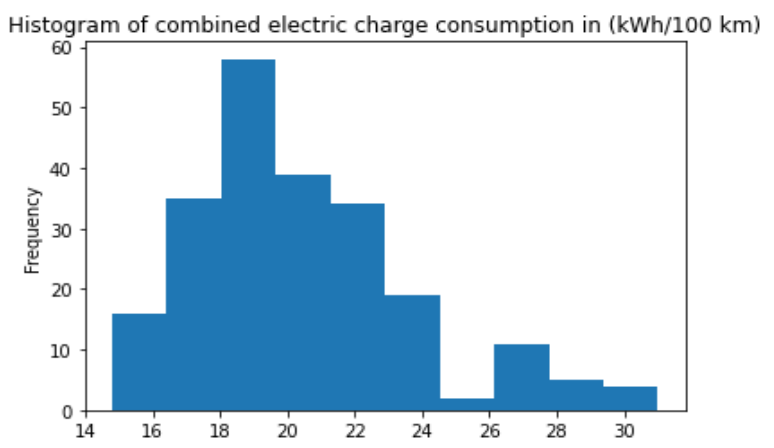

(b)

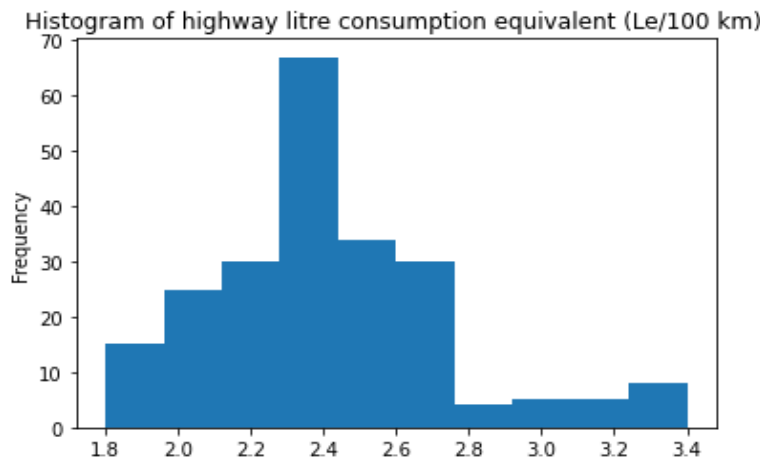

(d)

Histogram of combined Highway \& City litre consumption equivalent (Le/100 km)

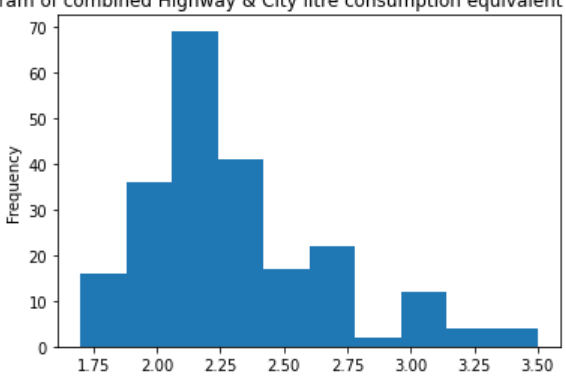

(f)

Figure 3. Cont. 


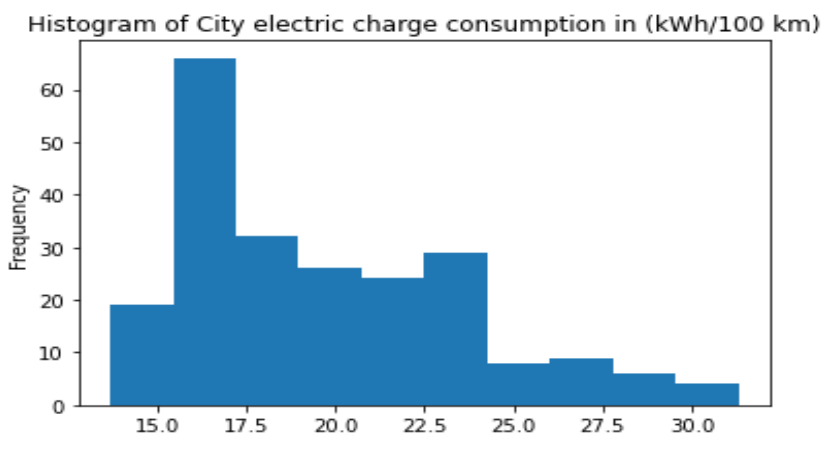

(g)

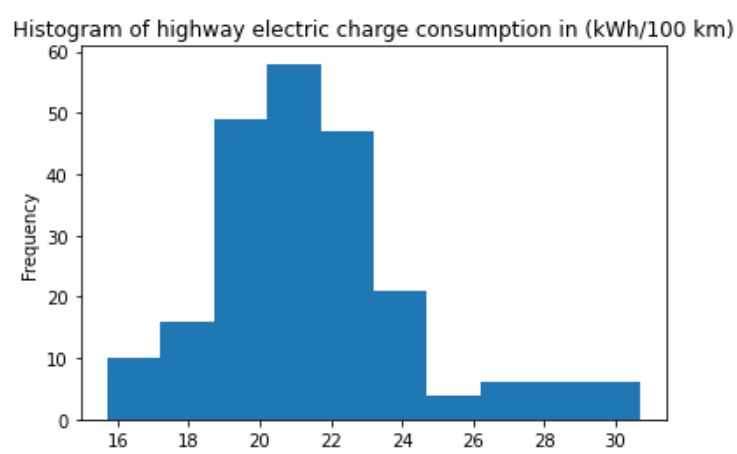

(h)

Range (the estimated driving distance (in kilometres) on a fully charged battery)

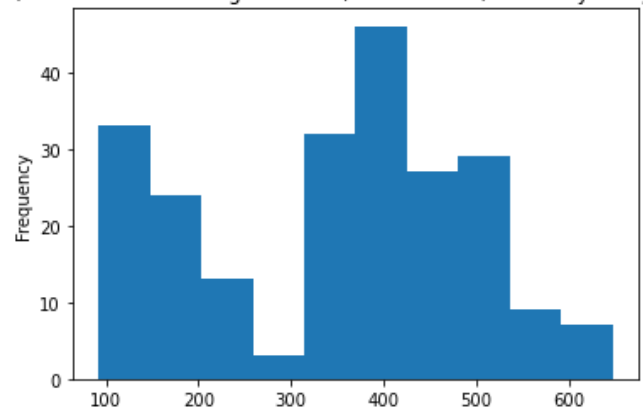

(i)

Figure 3. Dataset visualization. (a) Motor Power; (b) Combined electric charge consumption; (c) City litre consumption equivalent; (d) Highway litre consumption equivalent; (e) Recharge Time; (f) Combined (highway and city) consumption equivalent; (g) City electric charge consumption; (h) highway electric charge consumption; (i) Range.

\subsubsection{Formation of the Proposed Model for PEV}

To build or form the DANN model, a Python library known as Keras was imported and used for this process. The phases of model formation are described below.

\section{- Selection of Model Parameters}

Using eight variables as inputs and one as the target variable, and Keras library in Python, the model was built. The details of the model formation are shown in Figure 4 . The input layer consists of eight variables. It is represented by $\operatorname{dim}=8$. The 1 st hidden layer consists of 100 nodes, kernel initializer (normal), and activation function (ReLu). The duty of an activation function is to transform its inputs into outputs within a certain range. The 2nd hidden layer consists of 50 nodes, kernel initializer (normal), and activation function (tanh). Tanh activation function is a hyperbolic function. It takes the input as the real value and squash it to a range of -1 and +1 . Larger inputs are closer to 1 and smaller ones tend to -1 . Output layer consists of one node and a kernel initializer. The kernel initializer, which is normal of $\mathrm{x}$, takes the negative function as 0 .

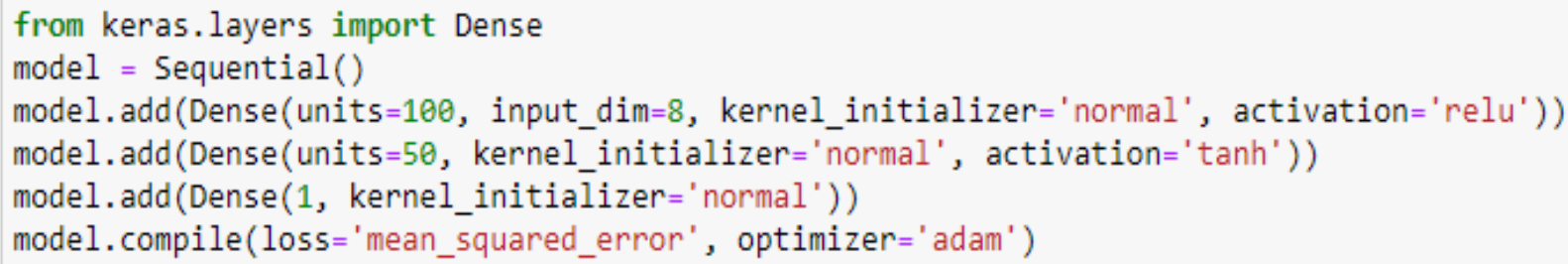

Figure 4. Selected parameters for the model. 


\section{- Compiling the Proposed Model}

Compiling involves specifying the parameters to measure the performance of the model. Model compiling is usually carried out before training. For compiling, regression loss function known as mean square error was employed. An optimizer called "Adam" in Keras library was used for the optimization process. Optimizers are algorithms for updating the weights of ANNs during the training process. Adam optimizer is currently the best optimizer for multilayer neural network. The optimizer in Keras operates on the principle of gradient descent algorithm. The systematic process of modifying or adjusting the weights is known as learning rules. Optimizer helps in solving the problem of hyperparameter tunning. There is no need of manually tuning the hyperparameter [25].

\section{- Training, Testing and Evaluation}

The Multilayer Perceptron (MLP) model was trained by calling the fit function. After training, the model was tested/validated by calling the fit function. The model was evaluated by calling the evaluation function.

\subsection{Design Fundamentals for the Model Parameters}

This section expounded more on the parameters selected for the model with respect to design.

\subsubsection{Determination of Electric Motor Power in $\mathrm{kW}$}

Powertrain motor (or electric motor) and battery pack are parts of the critical components of PEVs. Figure 5 shows a typical PEV and its components. For a vehicle on the ground floor, where the slope angle is zero, its air density is $1.25 \mathrm{~kg} \mathrm{~m}^{-3}$; the tractive force at the wheel is given by [28].

$$
\mathrm{F}=\mu \mathrm{mg}+0.625 \mathrm{AC}_{\mathrm{d}} \mathrm{V}^{2}+\mathrm{ma}+\mathrm{I} \frac{G^{2}}{\eta r^{2}} \mathrm{a}
$$

where: $\mathrm{m}=$ mass of the vehicle, $\mathrm{g}=$ acceleration due to gravity $\left(9.8 \mathrm{~m} / \mathrm{s}^{2}\right), \mu=$ Rolling resistance coefficient, $\mathrm{C}_{\mathrm{d}}=$ aerodynamic coefficient, $\mathrm{A}=$ frontal area in $\mathrm{m}^{2}, \mathrm{~V}=$ Vehicle speed, a = acceleration of the vehicle, $\mathrm{I}=$ moment of inertial, $r=$ radius of the tyre, $\eta=$ gear system efficiency, $G=$ gear ratio.

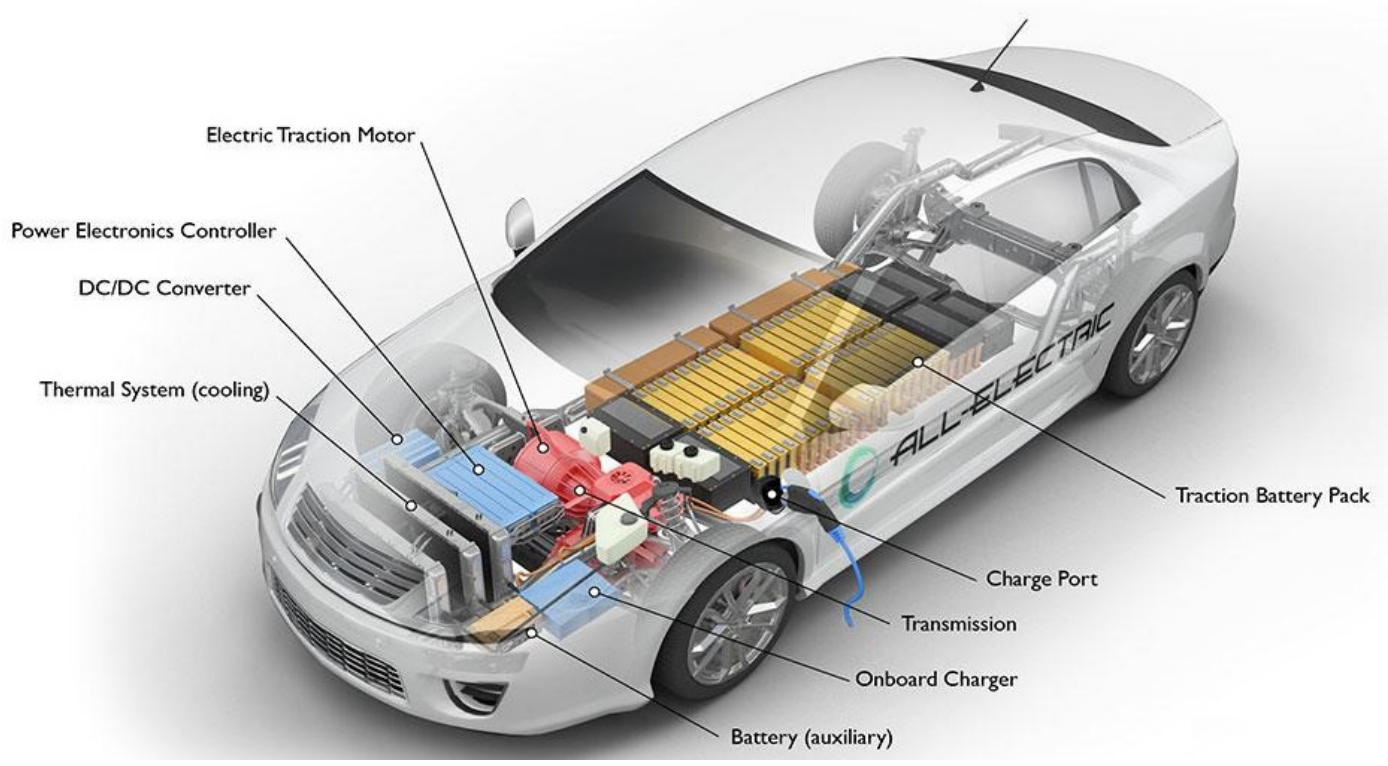

Figure 5. Pure electric vehicle [29]. 
The power of the electric motor is determined by multiplying the tractive force at the wheel by the velocity. It is given as,

$$
\mathrm{P}=\mathrm{FV}=\left[\mu \mathrm{mg}+0.625 \mathrm{AC}_{\mathrm{d}} \mathrm{V}^{2}+\mathrm{ma}+\mathrm{I} \frac{G^{2}}{\eta r^{2}} \mathrm{a}\right] \mathrm{V}
$$

It is important to know the power required at various speed to be able to design appropriately. This will also assist in determining the capacity of the electric motor needed to be selected at the design stage of PEVs. This is the reason for selecting electric motor power as a parameter for the model. The power available for the electric motor is also a function of the battery power or battery density. This makes battery recharge time an important factor.

\subsubsection{Determination of Fuel Economy in Pure Electric Vehicles}

The fuel economy (FE) of internal combustion engine vehicles is defined as the fuel consumption per km or miles per gallon. In the United States, the government agency assigned to regulate car fuel and emission related issues is known as Environmental Protection Agency (EPA). The agency has established fuel economy standard [30,31] figures for city driving, highway driving and combined cases. For the combined case, the figure fuel economy is $55 \%$ of city and $45 \%$ of highway, in MPG (miles per gallon).

$$
\mathrm{FE}_{\text {combined }}=\frac{1}{\frac{0.55}{\text { City fuel economy }}+\frac{0.45}{\text { highway fuel economy }}}
$$

For PEVs, the fuel economy is defined by electric consumption over certain range in $\mathrm{km}$.

$$
\text { Fuel Economy }=\frac{W h}{\text { mile }} \text { or } \mathrm{kWh} / 100 \mathrm{~km}
$$

One gallon of gasoline contains $33.7 \mathrm{kWh}$ [30].

One litre of gasoline contains the energy equivalent to $8.9 \mathrm{kWh}$ of electricity [2].

$$
\text { Fuel Economy gasoline equivalent }=\frac{1}{\mathrm{Wh} / \text { miles }} \times 33,700
$$

For this study, fuel economy figures were therefore considered as parts of the critical variables for the model. The following fuel economy figures were selected for this work: city electric charge consumption $(\mathrm{kWh} / 100 \mathrm{~km})$, highway electric charge consumption $(\mathrm{kWh} / 100 \mathrm{~km})$, combined electric charge consumption $(\mathrm{kWh} / 100 \mathrm{~km})$, city litre consumption equivalent $(\mathrm{Le} / 100 \mathrm{~km})$, highway litre consumption equivalent $(\mathrm{Le} / 100 \mathrm{~km})$, and combined litre consumption equivalent (Le/100 km).

\subsection{Redefining of Variables}

To further evaluate the efficiency of model, the selected nine variables were redefined randomly, and their predictions were obtained. The equations below were used to redefine the variables.

$$
\begin{aligned}
& \text { Mot, } V_{1}=\left(\frac{x}{2}\right)+5 \\
& \text { CE, } V_{2}=2 x-1 \\
& \text { HE, } V_{3}=2 x+5 \\
& \text { ComE, } V_{4}=2 x-3
\end{aligned}
$$




$$
\begin{gathered}
\text { CGEq, } V_{5}=x^{2}+8 \\
\text { HGEg, } V_{6}=x^{2} \\
\text { ComGEq, } V_{7}=2 x^{2}+2 \\
\text { Ra, } V_{8}=1.2 \frac{x}{2}+5 \\
\operatorname{ReT}, V_{9}=2 x-5
\end{gathered}
$$

\subsection{Proposed Model Evaluation Using Error Functions}

The model is also evaluated using the error functions such as mean absolute error (MAE), mean square error (MSE) and root mean square error (RMSE). The results are discussed in Section 4. Errors of the model were determined using combined electrical charge consumption.

$$
\begin{aligned}
\text { MAE } & =\frac{1}{n} \sum_{i=1}^{n}\left|Y_{\text {Predicted }(i)}-Y_{\text {desired }(i)}\right| \\
\text { MSE } & =\frac{1}{n}\left(\sum_{i=1}^{n}\left(Y_{\text {Predicted }(i)}-Y_{\text {desired }(i)}\right)^{2}\right) \\
\text { RMSE } & =\sqrt{\frac{1}{n}\left(\sum_{i=1}^{n}\left(Y_{\text {Predicted }(i)}-Y_{\text {desired }(i)}\right)^{2}\right)}
\end{aligned}
$$

\section{Results of the Predictions}

\subsection{Prediction of the Combined Electric Charge Consumption}

For the first prediction, the combined electric charge consumption datasets were fed into the box in Figure 1 as the target variable while all other eight variables were used as the inputs. After training and testing, the combined electric charge consumption was the predicted variable. Table $2 \mathrm{~b}$ shows a good predicting result for the combined electrical charge consumption. This is because the values of the errors are negligible by checking rows, when comparing the predicted output to the target variable in the last two columns. Table 2a shows the summary of the model with the number of parameters in each of the dense layers. The first dense layer has 900 trainable parameters. The second dense layer has 5050 trainable parameters. This is the layer that has the highest number of trainable parameters. The last dense layer has 51 trainable parameters. This layer has the lowest number of trainable parameters. The model had a total of 6001 trainable parameters. Figure $6 \mathrm{a}$ indicated that the profile of the predicted output perfectly overlapped with the target variable. The degree of overlap is between $90-96 \%$. The overall training and testing losses were $3.9132 \times 10^{-6}$ and $9.698 \times 10^{-7}$, respectively. This signifies a good degree of accuracy. Figure $6 \mathrm{~b}$ shows the behaviour of overall model loss as epoch increases. The testing error can be seen to be decreasing as the epoch increases from 0 to 1.0 epoch. It continued to decrease as epoch rises from 1.0 to 4.0. The training error was almost constant when epoch rises from 0 to 1.5 epoch. It was found to be decreasing as epoch rises from 1.5 to 4.0 epoch. 
Table 2. Prediction result at model formation.

\begin{tabular}{ccc}
\hline & (a) Summary of the Model & \\
\hline & Model Summary & Parameter \# \\
\hline Layer (type) & Output Shape & 900 \\
\hline Dense_33 (Dense) & (None, 100) & 5050 \\
Dense_34 (Dense) & (None, 50) & 51 \\
Dense_35 (Dense) & (None, 1) & \\
Total parameters: & 6001 & \\
Total parameters: & 0001 & \\
Non-trainableparameters: & 0
\end{tabular}

(b) Predicted Combined Electric Charge Consumption

\begin{tabular}{ccccccccccc}
\hline & CE & ReT & HE & CGEq & HGEq & ComGEq & Mot & Ra & ComE & PredictedComE \\
\hline 0 & 23.8 & 12 & 23.2 & 2.7 & 2.6 & 2.6 & 270 & 426 & 23.6 & 23.518833 \\
1 & 16.2 & 5 & 19.7 & 1.8 & 2.2 & 2 & 125 & 183 & 17.8 & 17.820225 \\
2 & 18.7 & 3 & 23.1 & 2.1 & 2.6 & 2.3 & 60 & 92 & 20.7 & 20.755283 \\
3 & 14.5 & 5.8 & 17.4 & 1.6 & 1.9 & 1.8 & 100 & 274 & 15.8 & 15.946842 \\
4 & 18.2 & 10 & 19.8 & 2 & 2.2 & 2.1 & 358 & 488 & 18.9 & 19.017338 \\
5 & 16.8 & 5 & 22.4 & 1.9 & 2.5 & 2.2 & 81 & 179 & 19.3 & 19.458347 \\
6 & 17 & 6 & 20.7 & 1.9 & 2.3 & 2.1 & 80 & 172 & 18.6 & 18.737461 \\
7 & 16.8 & 5.3 & 18.6 & 1.9 & 2.1 & 2 & 100 & 201 & 17.4 & 17.652714 \\
8 & 16.3 & 10 & 18.7 & 1.8 & 2.1 & 2 & 358 & 507 & 17.4 & 17.585972 \\
9 & 19.9 & 8.8 & 22.4 & 2.2 & 2.5 & 2.4 & 198 & 370 & 21 & 21.097385 \\
\hline
\end{tabular}

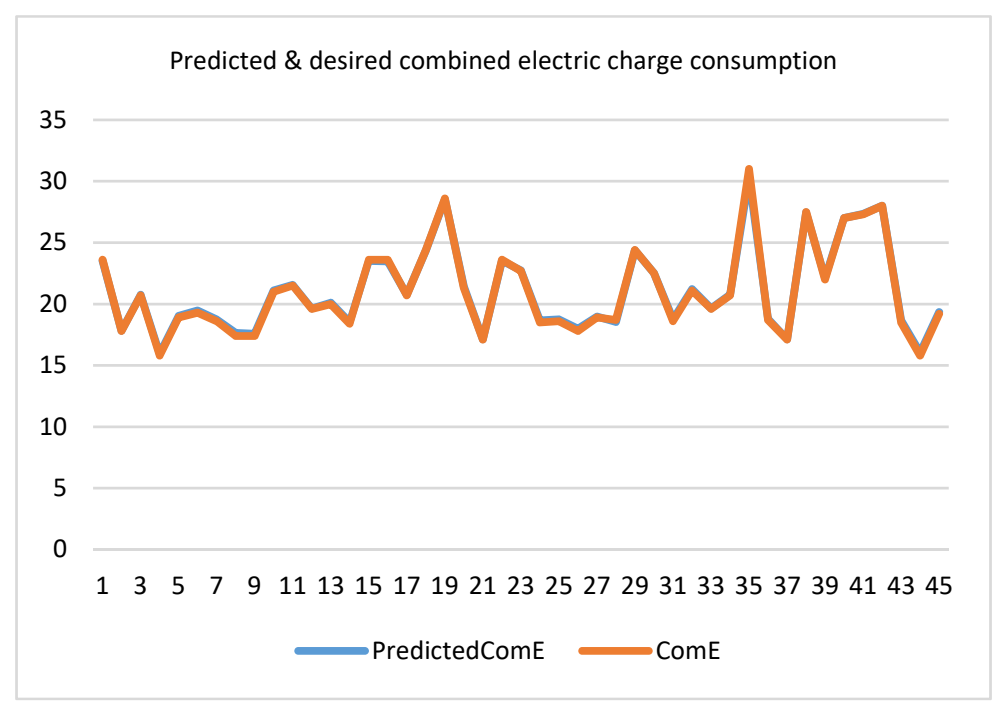

(a)

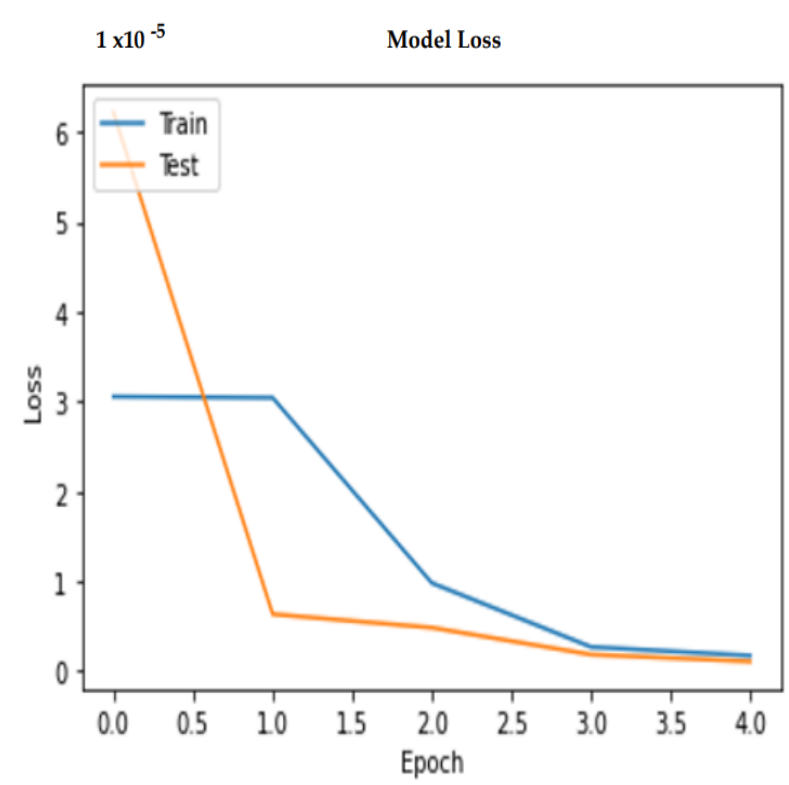

(b)

Figure 6. Performance measurement of the combined electric charge consumption. (a) Behaviour of the combined electric charge consumption; (b) Model loss with increase in epoch.

After the prediction of the combined electric charge consumption, the model was equally used to predict the remaining eight variables.

\subsection{Prediction of the City Electric Charge Consumption (CE)}

Using the same single-output model in Figure 4, the city electric charge was used as the target variable while the other 8 variables were fed into the model as the inputs. The overall error for the model during training and testing were 0.0071 and 0.0021 , respectively. 
Table 3 shows that the unit errors are negligible by comparing the last two columns on the right-hand side to each other. Figure 7a shows that both the target and the predicted variables overlapped. The degree of overlap is between $90-95 \%$. It can be attested that the prediction was of high degree of accuracy. Figure $7 \mathrm{~b}$ indicated how the training error was always changing and decreasing as the number of epochs increases. However, the testing error increases as epoch rises from 0 to 1.0. It began to decrease as epoch rises from 1.0 to 3.0 epoch. The testing error was found to be increasing as epoch rises from 3.0 to 4.0 epoch.

Table 3. Predicted and desired city electric charge consumption.

\begin{tabular}{ccccccccccc}
\hline & ReT & HE & CGEq & HGEq & ComGEq & Mot & Ra & ComE & CE & Predicted CE \\
\hline 0 & 12 & 23.2 & 2.7 & 2.6 & 2.6 & 270 & 426 & 23.6 & 23.8 & 24.129328 \\
1 & 5 & 19.7 & 1.8 & 2.2 & 2 & 125 & 183 & 17.8 & 16.2 & 16.105284 \\
2 & 3 & 23.1 & 2.1 & 2.6 & 2.3 & 60 & 92 & 20.7 & 18.7 & 18.772221 \\
3 & 5.8 & 17.4 & 1.6 & 1.9 & 1.8 & 100 & 274 & 15.8 & 14.5 & 14.315234 \\
4 & 10 & 19.8 & 2 & 2.2 & 2.1 & 358 & 488 & 18.9 & 18.2 & 18.061446 \\
5 & 5 & 22.4 & 1.9 & 2.5 & 2.2 & 81 & 179 & 19.3 & 16.8 & 16.890638 \\
6 & 6 & 20.7 & 1.9 & 2.3 & 2.1 & 80 & 172 & 18.6 & 17 & 16.919226 \\
7 & 5.3 & 18.6 & 1.9 & 2.1 & 2 & 100 & 201 & 17.4 & 16.8 & 16.77796 \\
8 & 10 & 18.7 & 1.8 & 2.1 & 2 & 358 & 507 & 17.4 & 16.3 & 16.444208 \\
9 & 8.8 & 22.4 & 2.2 & 2.5 & 2.4 & 198 & 370 & 21 & 19.9 & 19.894707 \\
\hline
\end{tabular}

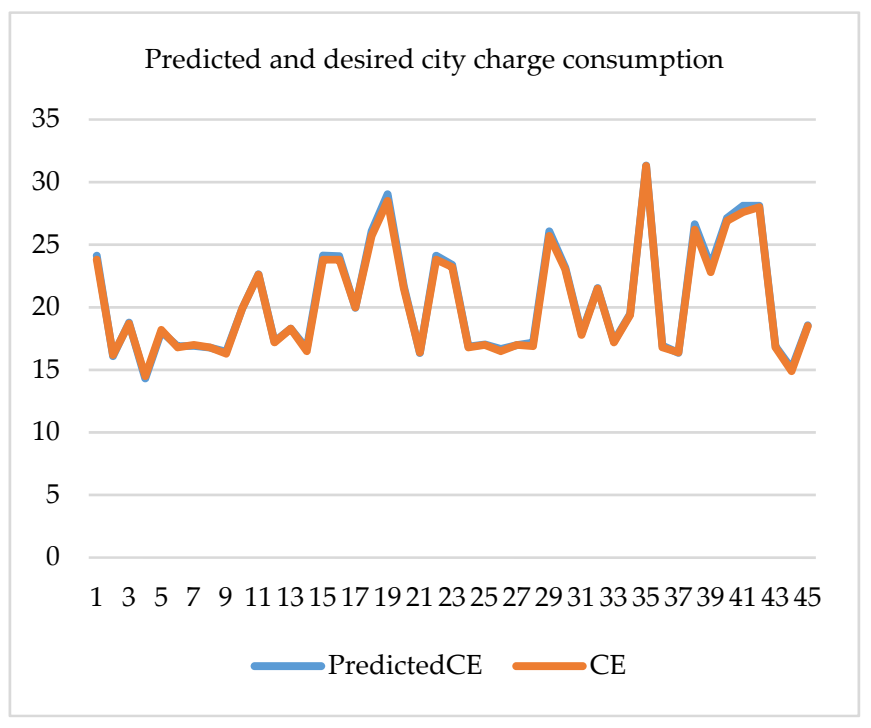

(a)

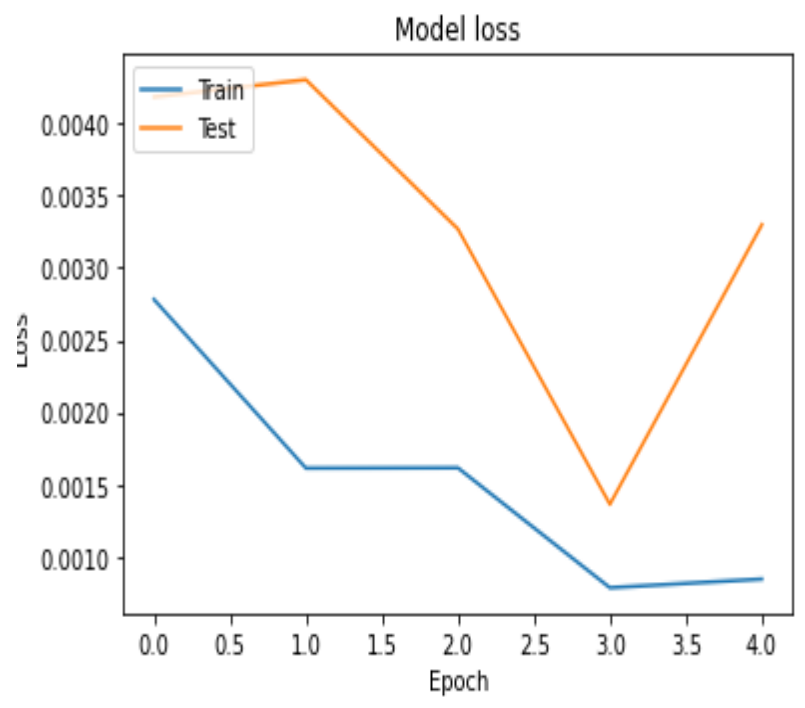

(b)

Figure 7. Predicted city electric charge consumption analysis. (a) Behaviour of the city electric charge consumption; (b) Model loss with increase in epoch.

\subsection{Prediction of the Recharge Time (ReT)}

For the prediction of the recharge time, 0.0011 and 0.0058932 are the model's training and testing losses. Figure $8 \mathrm{~b}$ shows the changes in the model loss for the training and testing as epoch increases. The losses were not uniform for both the training and the testing. The testing error decreases as the epoch increases to a value of 2.0. The testing error then begins to increase as the epoch rises from 2.0 to 3.0. The last two columns of Table 4 show the details of the predicted and desired recharge times. Unit errors along the rows were minimal in Table 4. Figure $8 \mathrm{a}$ also shows a good overlap between the predicted and the desired variables. The degree of overlap is between $90-96 \%$. The prediction was of high level of performance and precision. 


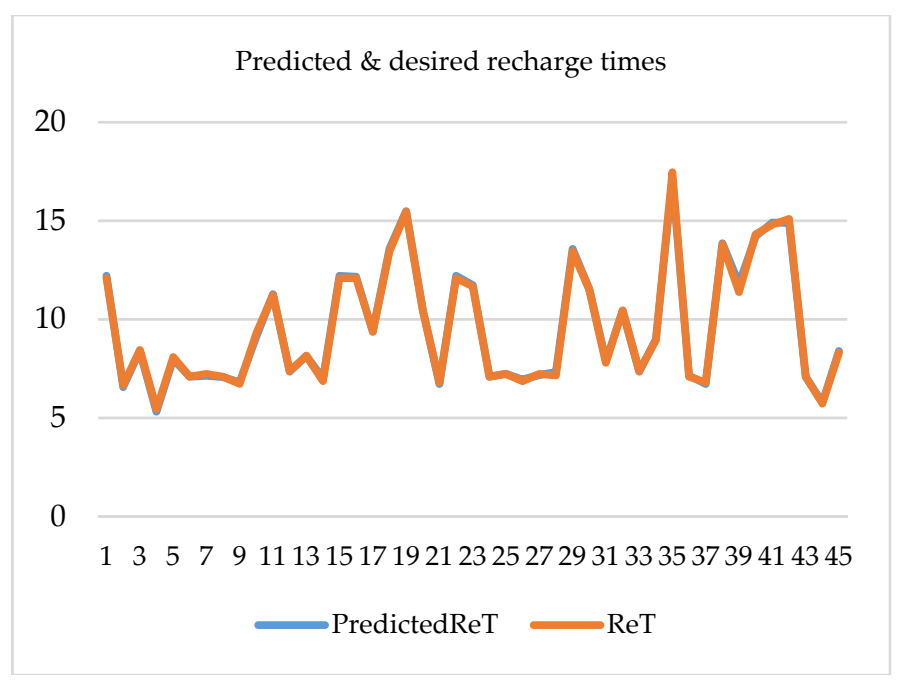

(a)

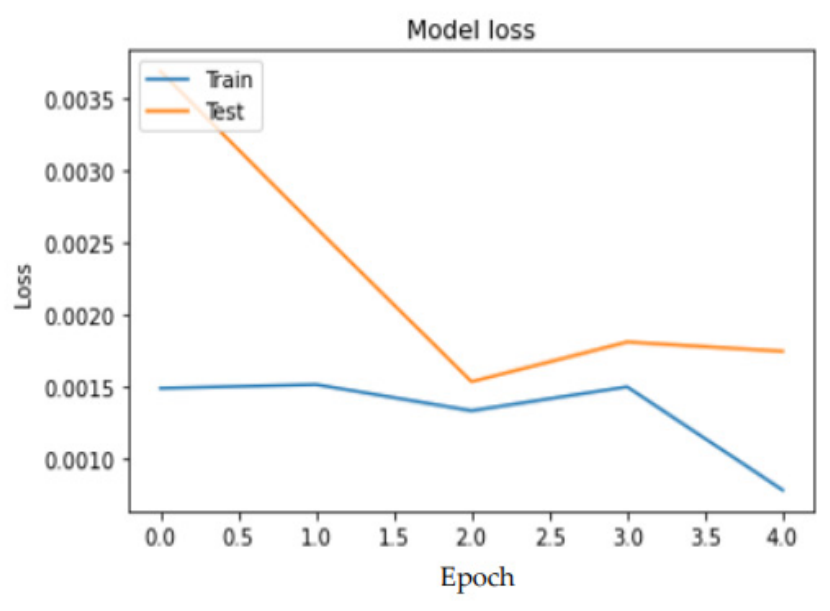

(b)

Figure 8. Predicted and desired recharge time analysis. (a) Behaviour of the predicted and desired of recharge time; (b) Model loss with increase in epoch.

Table 4. Predicted and desired Recharge Time.

\begin{tabular}{ccccccccccc}
\hline & HE & CGEq & HGEq & ComGEq & Mot & Ra & ComE & CE & ReT & Predicted ReT \\
\hline 0 & 24.28 & 2.49 & 2.75 & 2.53 & 402.63 & 347.18 & 22.28 & 23.43 & 12.099 & 12.21354 \\
1 & 17.12 & 1.96 & 2.10 & 2.068 & 135.04 & 217.75 & 16.82 & 16.55 & 6.67 & 6.568537 \\
2 & 15.06 & 2.48 & 2.32 & 2.53 & 268.84 & 159.723 & 14.78 & 19.99 & 8.45 & 8.39825 \\
3 & 17.93 & 1.603 & 1.95 & 1.72 & 45.847 & 195.43 & 18.87 & 14.17 & 5.45 & 5.329853 \\
4 & 22.227 & 1.971 & 2.24 & 2.068 & 179.64 & 425.73 & 23.67 & 17.85 & 8.096 & 7.97622 \\
5 & 17.1 & 2.37 & 2.17 & 2.41 & 224.23 & 178.468 & 16.73 & 18.32 & 7.094 & 7.096871 \\
6 & 18.13 & 2.1 & 2.17 & 2.18 & 179.64 & 177.58 & 16.58 & 17.5 & 7.24 & 7.139585 \\
7 & 17.42 & 1.79 & 2.17 & 1.95 & 135.04 & 195.43 & 17.22 & 16.072 & 7.09 & 7.081111 \\
8 & 22.23 & 1.8 & 2.1 & 1.95 & 135.04 & 425.73 & 24.1 & 16.072 & 6.74 & 6.821907 \\
9 & 20.1 & 2.37 & 2.39 & 2.41 & 313.44 & 282.9 & 21.02 & 20.34 & 9.31 & 9.131731 \\
\hline
\end{tabular}

\subsection{Prediction of the Highway Electric Charge Consumption}

The highway electric charge consumption was made the target variable in this case while all other selected parameters became the inputs. 0.0013 was the overall model training error while 0.0011 was the loss value for testing. The model structure is the same for this prediction as shown in Figure 4. Table 5 shows the predicted output and desired highway electric charge consumption in the last two columns, on the right-hand side. Absolute errors are of small values by comparing the elements in the rows of the predicted and the desire variables. Figure 9 a shows the excellent overlap of the target and the predicted variables. The degree of overlap is between $90-97 \%$. Figure $9 \mathrm{~b}$ also shows how the model loss changes with epoch during the training and the validation stages. The model losses were not constant. Both the testing and the training errors began to increase in values as epoch rises from 0 to 1.0. The testing and training errors then began to decrease in values as epoch rises from 1.0 to 3.0. The training error later decreases in value while the testing error remains constant as the epoch increases from 3.0 to 4.0 epoch. Of course, the prediction is of an acceptable degree of accuracy. 
Table 5. Predicted and desired highway electric charge consumption.

\begin{tabular}{cccccccccc}
\hline CGEq & HGEq & ComGEq & Mot & Ra & ComE & CE & ReT & HE & Predicted HE \\
\hline 2.7 & 2.6 & 2.6 & 270 & 426 & 23.6 & 23.8 & 12 & 23.2 & 23.189518 \\
1.8 & 2.2 & 2 & 125 & 183 & 17.8 & 16.2 & 5 & 19.7 & 19.625205 \\
2.1 & 2.6 & 2.3 & 60 & 92 & 20.7 & 18.7 & 3 & 23.1 & 23.005917 \\
1.6 & 1.9 & 1.8 & 100 & 274 & 15.8 & 14.5 & 5.8 & 17.4 & 17.441454 \\
2 & 2.2 & 2.1 & 358 & 488 & 18.9 & 18.2 & 10 & 19.8 & 19.8613 \\
1.9 & 2.5 & 2.2 & 81 & 179 & 19.3 & 16.8 & 5 & 22.4 & 22.27434 \\
1.9 & 2.3 & 2.1 & 80 & 172 & 18.6 & 17 & 6 & 20.7 & 20.554855 \\
1.9 & 2.1 & 2 & 100 & 201 & 17.4 & 16.8 & 5.3 & 18.6 & 18.633545 \\
1.8 & 2.1 & 2 & 358 & 507 & 17.4 & 16.3 & 10 & 18.7 & 18.787008 \\
2.2 & 2.5 & 2.4 & 198 & 370 & 21 & 19.9 & 8.8 & 22.4 & 22.373301 \\
\hline
\end{tabular}

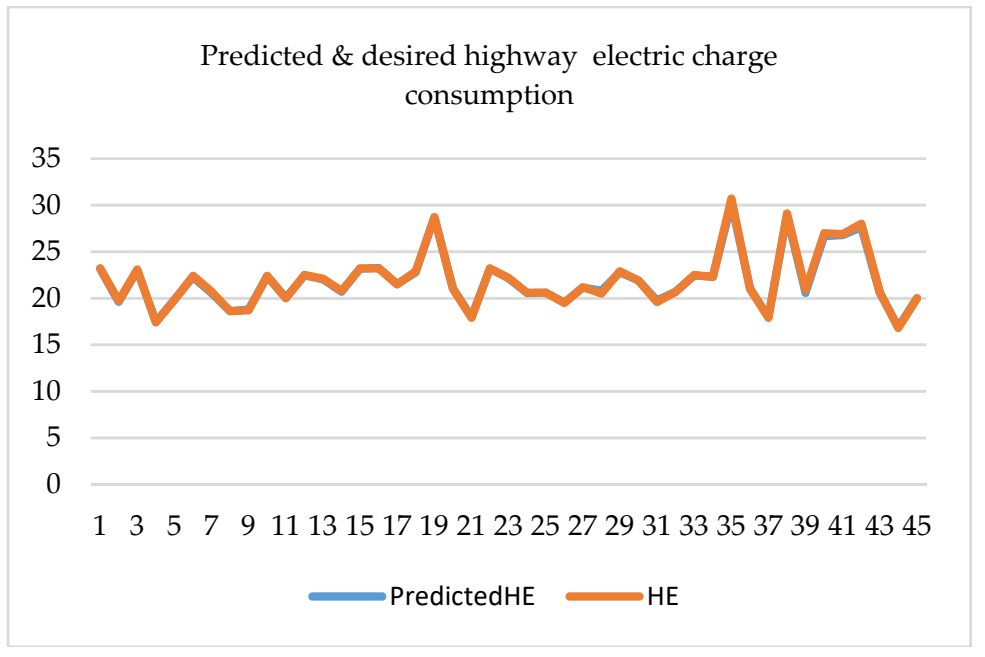

(a)

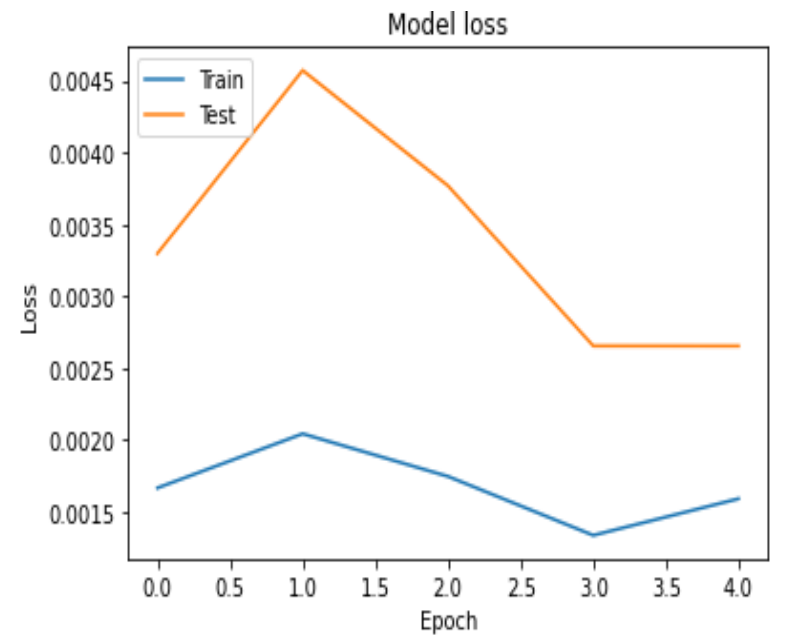

(b)

Figure 9. Predicted highway electric charge consumption analysis. (a) Behaviour of the highway electric charge consumption; (b) Model loss with increase in epoch.

\subsection{Prediction of the City Gasoline Litre Consumption Equivalent(CGEq)}

The single-output model was also used to predict the city gasoline litre consumption equivalent, and the predicted output variable is shown in Table 6 and Figure 10a. From the tabulated result, unit errors were negligible by comparing the predicted output to desired variable in Table 6. The graphical profile in Figure 10a shows that errors were kept to a minimal level. The degree of overlap between the predicted and the desired variables was exceptional. The degree of overlap is between $90-96 \%$. This is a measure of precision and accuracy. Figure $10 \mathrm{~b}$ shows the profile of the model losses during testing and training phases. The training error increases in value as epoch rises from 0 to 2.0; it then continues to decrease. The testing error can be seen increasing as epoch rises from 0 to 1.5. The model testing error was found to be decreasing in value as epoch increases from 1.5 to 2.0. Its increases in value as epoch rises from 2.0 to 3.0. The testing error experiences decrease in value as epoch increases from 3.0 to 4.0 . 
Table 6. Predicted and desired city gasoline litre consumption equivalent.

\begin{tabular}{ccccccccccc}
\hline & HGEq & ComGEq & Mot & Ra & ComE & CE & ReT & HE & CGEq & Predicted CGEq \\
\hline 0 & 2.6 & 2.6 & 270 & 426 & 23.6 & 23.8 & 12 & 23.2 & 2.7 & 2.683975 \\
1 & 2.2 & 2 & 125 & 183 & 17.8 & 16.2 & 5 & 19.7 & 1.8 & 1.821692 \\
2 & 2.6 & 2.3 & 60 & 92 & 20.7 & 18.7 & 3 & 23.1 & 2.1 & 2.106232 \\
3 & 1.9 & 1.8 & 100 & 274 & 15.8 & 14.5 & 5.8 & 17.4 & 1.6 & 1.604526 \\
4 & 2.2 & 2.1 & 358 & 488 & 18.9 & 18.2 & 10 & 19.8 & 2 & 2.046841 \\
5 & 2.5 & 2.2 & 81 & 179 & 19.3 & 16.8 & 5 & 22.4 & 1.9 & 1.91557 \\
6 & 2.3 & 2.1 & 80 & 172 & 18.6 & 17 & 6 & 20.7 & 1.9 & 1.913931 \\
7 & 2.1 & 2 & 100 & 201 & 17.4 & 16.8 & 5.3 & 18.6 & 1.9 & 1.85855 \\
8 & 2.1 & 2 & 358 & 507 & 17.4 & 16.3 & 10 & 18.7 & 1.8 & 1.843146 \\
9 & 2.5 & 2.4 & 198 & 370 & 21 & 19.9 & 8.8 & 22.4 & 2.2 & 2.259193 \\
\hline
\end{tabular}

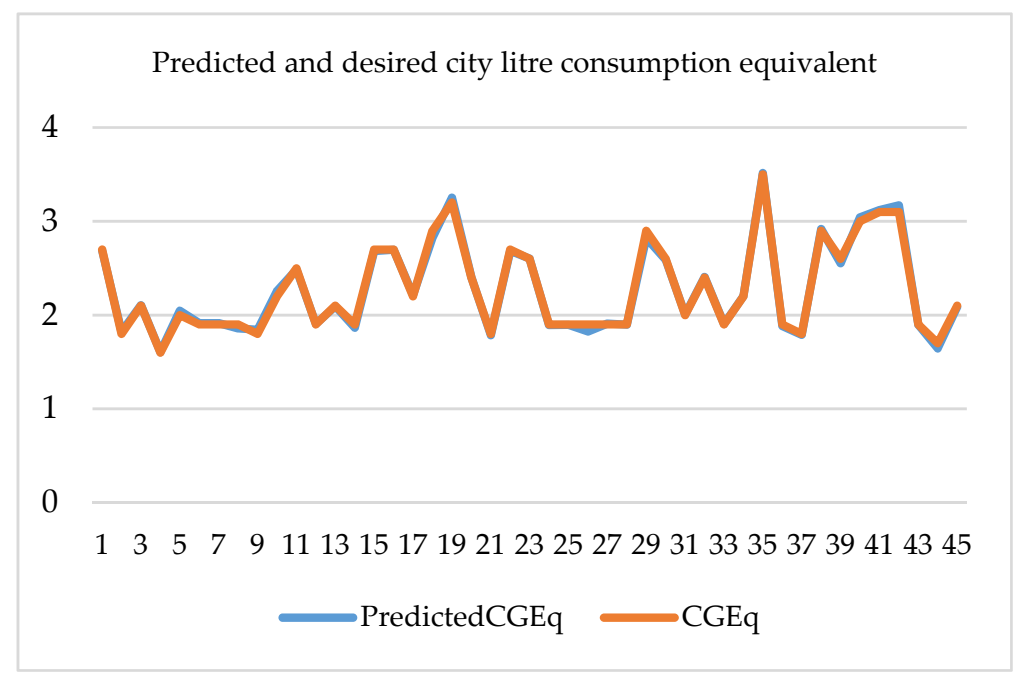

(a)

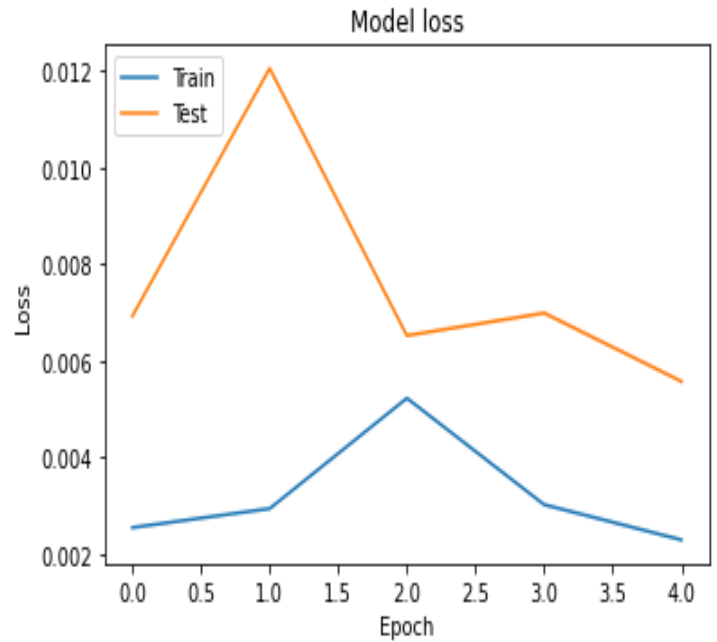

(b)

Figure 10. City gasoline litre consumption equivalent analysis. (a) Behaviour of city gasoline litre consumption equivalent; (b) Model loss with increase in epoch.

\subsection{Prediction of the Highway Gasoline Litre Consumption Equivalent (HGEq)}

Highway litre consumption was made the target variable and all other variables were used as the input values using the same single-output model already built. Overall training loss was 0.0039 while overall testing error was 0.0045 . Table 7 comparatively indicated that the absolute errors were negligible by considering the predicted and the target variables. Figure 11a also shows a high degree of overlap between the predicted and target variables. The degree of overlap is between $90-97 \%$. Figure $11 \mathrm{~b}$ shows the fluctuating profile of model loss for the training and the testing phases. The model testing error decreases as epoch increases 0 to 2.0. It increases as epoch rises from 2.0 to 3.0. It began to decrease as epoch increases from 3.0 to 4.0. The training error reduces in value as epoch increases from 0 to 1.0. It then begins to increase in value as the epoch rises from 1.0 to 2.0. It continues to fluctuate as epoch increases. The overall model losses attested that the model is good for the prediction of highway gasoline litre consumption equivalent. 
Table 7. Predicted and desired highway gasoline litre consumption equivalent.

\begin{tabular}{ccccccccccc}
\hline & ComGEq & Mot & Ra & ComE & CE & ReT & HE & CGEq & HGEq & Predicted HGEq \\
\hline 0 & 2.6 & 270 & 426 & 23.6 & 23.8 & 12 & 23.2 & 2.7 & 2.6 & 2.616106 \\
1 & 2 & 125 & 183 & 17.8 & 16.2 & 5 & 19.7 & 1.8 & 2.2 & 2.19862 \\
2 & 2.3 & 60 & 92 & 20.7 & 18.7 & 3 & 23.1 & 2.1 & 2.6 & 2.605645 \\
3 & 1.8 & 100 & 274 & 15.8 & 14.5 & 5.8 & 17.4 & 1.6 & 1.9 & 1.899809 \\
4 & 2.1 & 358 & 488 & 18.9 & 18.2 & 10 & 19.8 & 2 & 2.2 & 2.210691 \\
5 & 2.2 & 81 & 179 & 19.3 & 16.8 & 5 & 22.4 & 1.9 & 2.5 & 2.21091 \\
6 & 2.1 & 80 & 172 & 18.6 & 17 & 6 & 20.7 & 1.9 & 2.3 & 2.523738 \\
7 & 2 & 100 & 201 & 17.4 & 16.8 & 5.3 & 18.6 & 1.9 & 2.1 & 2.313696 \\
8 & 2 & 358 & 507 & 17.4 & 16.3 & 10 & 18.7 & 1.8 & 2.1 & 2.072292 \\
9 & 2.4 & 198 & 370 & 21 & 19.9 & 8.8 & 22.4 & 2.2 & 2.5 & 2.484709 \\
\hline
\end{tabular}

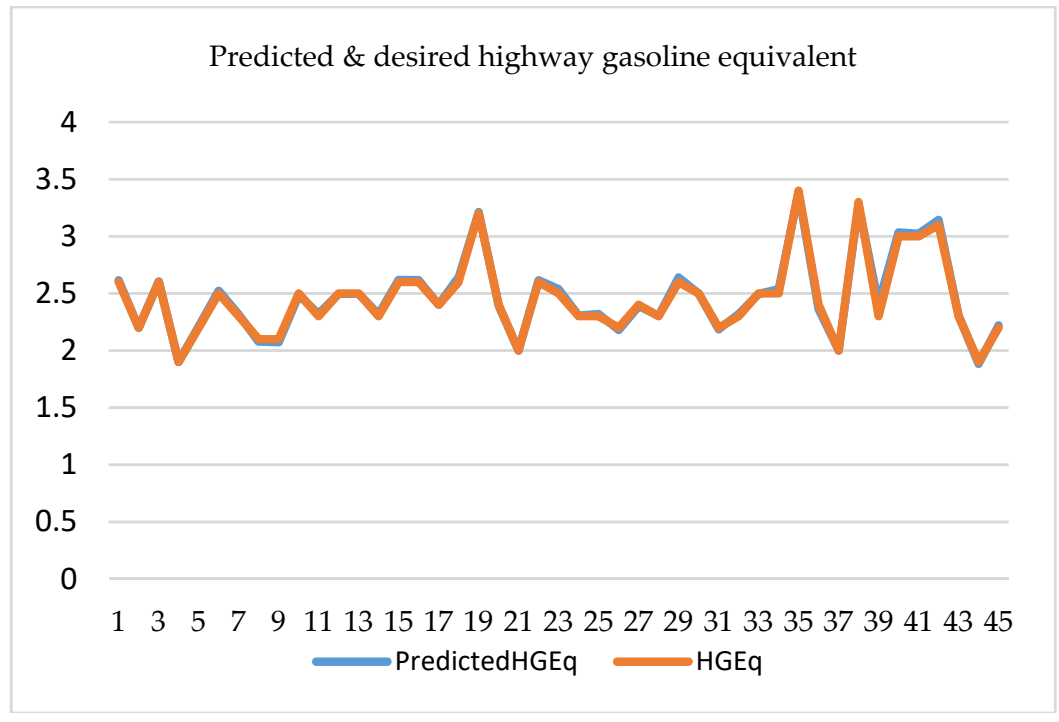

(a)

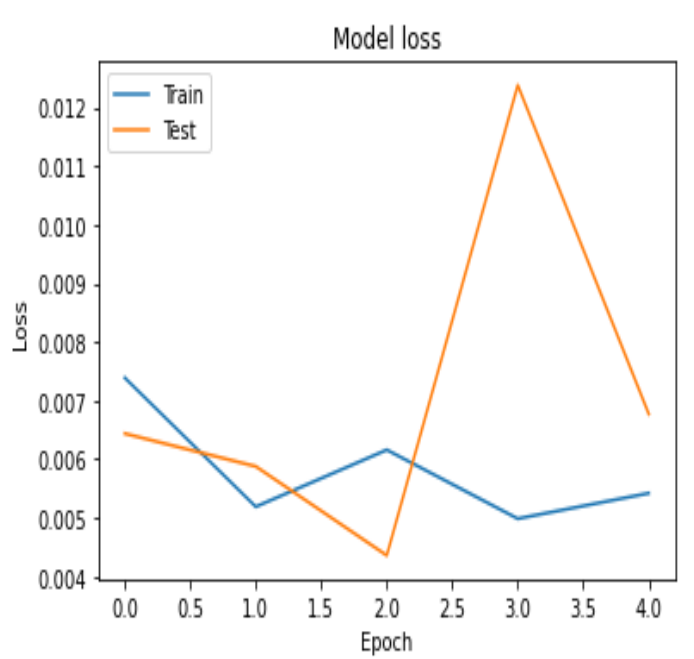

(b)

Figure 11. Highway gasoline litre consumption equivalent analysis. (a) Behaviour of the highway litre consumption equivalent; (b) Model loss with increase in epoch.

\subsection{Prediction of the Combined Gasoline Litre Consumption Equivalent}

Using the same single-output model of PEVs to predict the combined litre consumption equivalent, the results were analytically acceptable. Overall losses for the training and testing were 0.0017 and 0.0016 . Table 8 shows a minimized range of errors when the predicted and the desired variables in the last two columns are compared. Figure 12a did show that the prediction of the combined litre consumption equivalent was with high degree of accuracy, by comparing it with the desired variable, due to the degree of overlap between their profiles. The degree of overlap is between $90-97 \%$. From Figure 12b, the value of the model loss for the training was almost uniform while that of the testing varied as epoch rises. 
Table 8. Predicted and desired combined gasoline litre consumption equivalent.

\begin{tabular}{ccccccccccc}
\hline & Mot & Ra & ComE & CE & ReT & HE & CGEq & HGEq & ComGEq & Predicted ComGEq \\
\hline 0 & 270 & 426 & 23.6 & 23.8 & 12 & 23.2 & 2.7 & 2.6 & 2.6 & 2.59812 \\
1 & 125 & 183 & 17.8 & 16.2 & 5 & 19.7 & 1.8 & 2.2 & 2 & 2.02092 \\
2 & 60 & 92 & 20.7 & 18.7 & 3 & 23.1 & 2.1 & 2.6 & 2.3 & 2.305117 \\
3 & 100 & 274 & 15.8 & 14.5 & 5.8 & 17.4 & 1.6 & 1.9 & 1.8 & 1.796104 \\
4 & 358 & 488 & 18.9 & 18.2 & 10 & 19.8 & 2 & 2.2 & 2.1 & 2.140992 \\
5 & 81 & 179 & 19.3 & 16.8 & 5 & 22.4 & 1.9 & 2.5 & 2.2 & 2.197978 \\
6 & 80 & 172 & 18.6 & 17 & 6 & 20.7 & 1.9 & 2.3 & 2.1 & 2.11947 \\
7 & 100 & 201 & 17.4 & 16.8 & 5.3 & 18.6 & 1.9 & 2.1 & 2 & 1.998774 \\
8 & 358 & 507 & 17.4 & 16.3 & 10 & 18.7 & 1.8 & 2.1 & 2 & 1.95528 \\
9 & 198 & 370 & 21 & 19.9 & 8.8 & 22.4 & 2.2 & 2.5 & 2.4 & 2.36263 \\
\hline
\end{tabular}

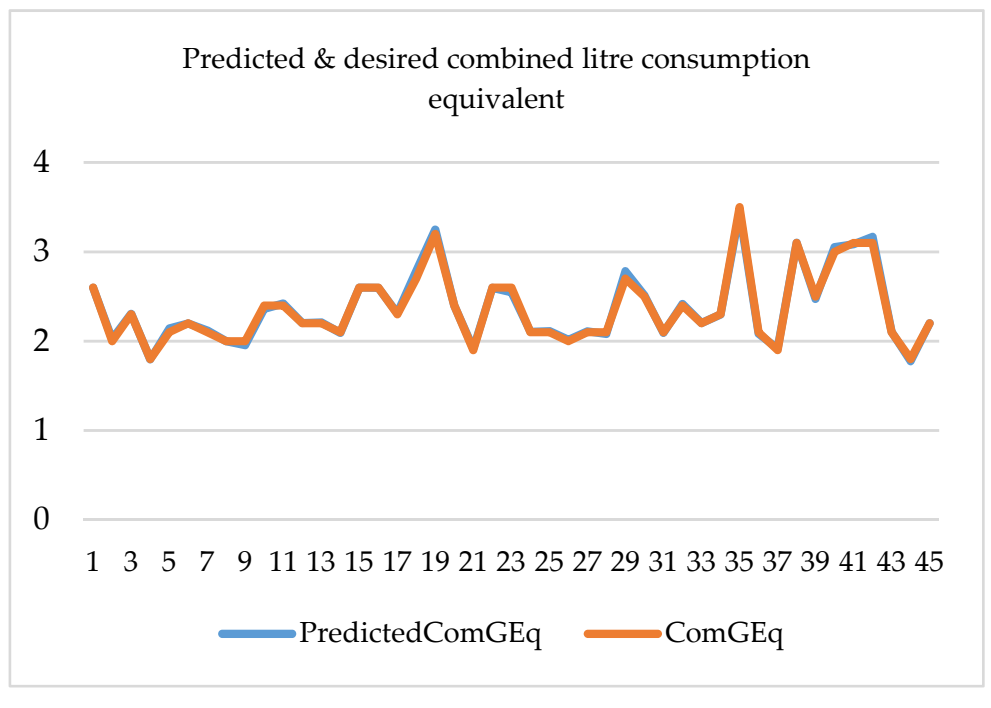

(a)

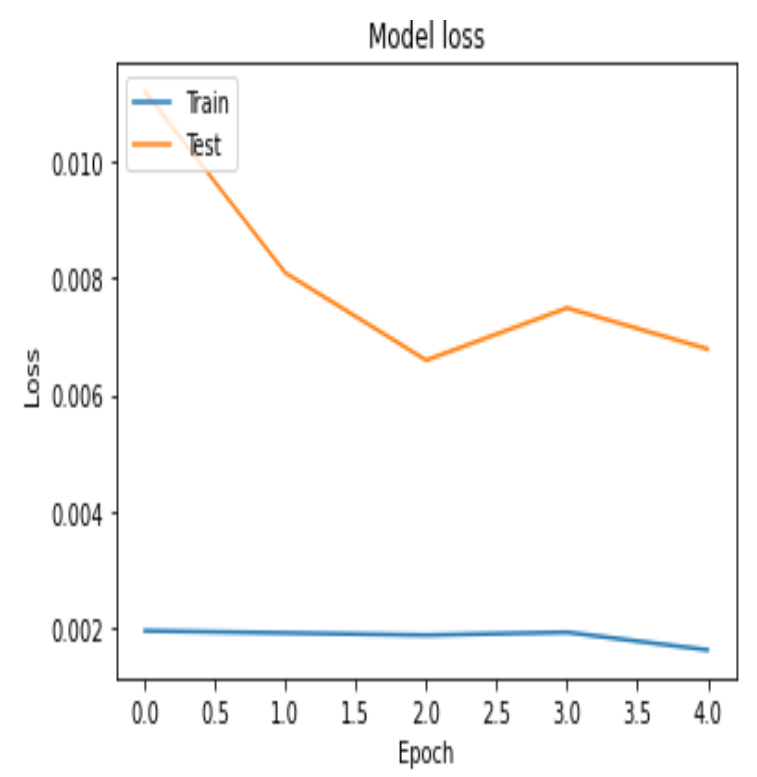

(b)

Figure 12. Combined gasoline litre consumption equivalent analysis. (a) Behaviour of the combined litre consumption equivalent; (b) Model loss with increase in epoch.

\subsection{Prediction of the Electrical Motor Power (Mot)}

Electric motor power was equally set as a target variable while all other variables were used as the inputs using the single-output model. The system was trained and validated. The predicted variable was observed. The overall model losses for the training and testing were 0.0181 and 0.0134 , respectively. Table 9 shows the predicted and the desired electrical motor power. Figure 13a shows that the degree of overlap between the predicted and the target variable. The degree of overlap is between $75-85 \%$. Figure $13 \mathrm{~b}$ shows changes in model losses as epoch increases. 
Table 9. Predicted and desired electrical motor power.

\begin{tabular}{ccccccccccc}
\hline & Ra & ComE & CE & ReT & HE & CGEq & HGEq & ComGEq & Mot & Predicted Mot \\
\hline 0 & 426 & 23.6 & 23.8 & 12 & 23.2 & 2.7 & 2.6 & 2.6 & 270 & 325.39328 \\
1 & 183 & 17.8 & 16.2 & 5 & 19.7 & 1.8 & 2.2 & 2 & 125 & 127.758652 \\
2 & 92 & 20.7 & 18.7 & 3 & 23.1 & 2.1 & 2.6 & 2.3 & 60 & 61.349003 \\
3 & 274 & 15.8 & 14.5 & 5.8 & 17.4 & 1.6 & 1.9 & 1.8 & 100 & 121.266022 \\
4 & 488 & 18.9 & 18.2 & 10 & 19.8 & 2 & 2.2 & 2.1 & 358 & 390.781677 \\
5 & 179 & 19.3 & 16.8 & 5 & 22.4 & 1.9 & 2.5 & 2.2 & 81 & 87.229782 \\
6 & 172 & 18.6 & 17 & 6 & 20.7 & 1.9 & 2.3 & 2.1 & 80 & 86.093201 \\
7 & 201 & 17.4 & 16.8 & 5.3 & 18.6 & 1.9 & 2.1 & 2 & 100 & 110.93045 \\
8 & 507 & 17.4 & 16.3 & 10 & 18.7 & 1.8 & 2.1 & 2 & 358 & 350.521454 \\
9 & 370 & 21 & 19.9 & 8.8 & 22.4 & 2.2 & 2.5 & 2.4 & 198 & 218.790649 \\
\hline
\end{tabular}

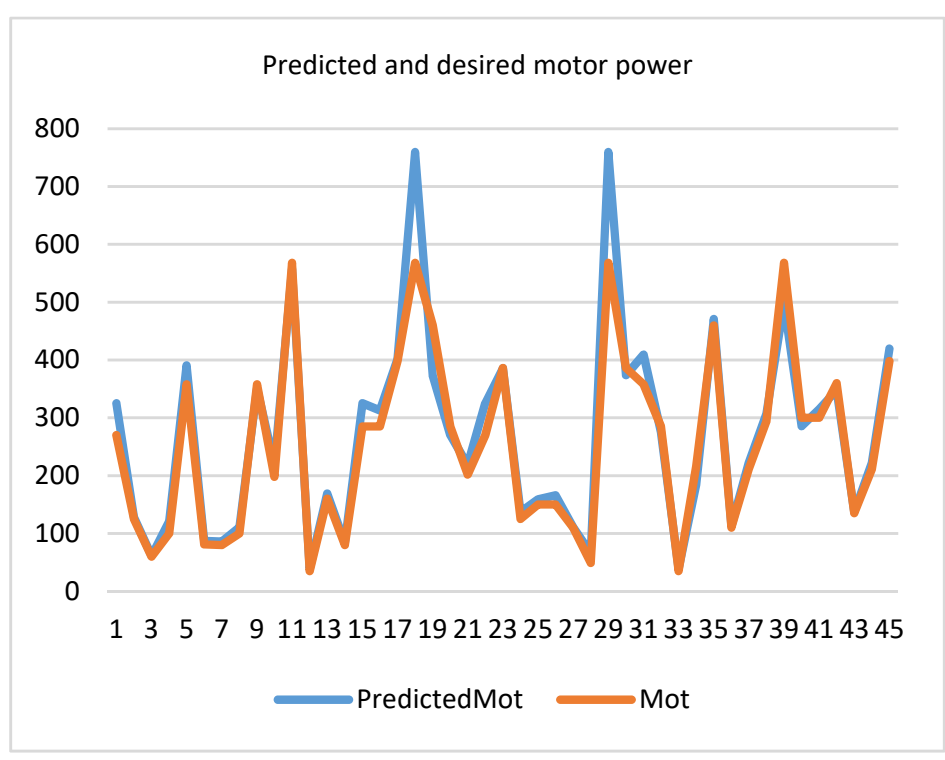

(a)

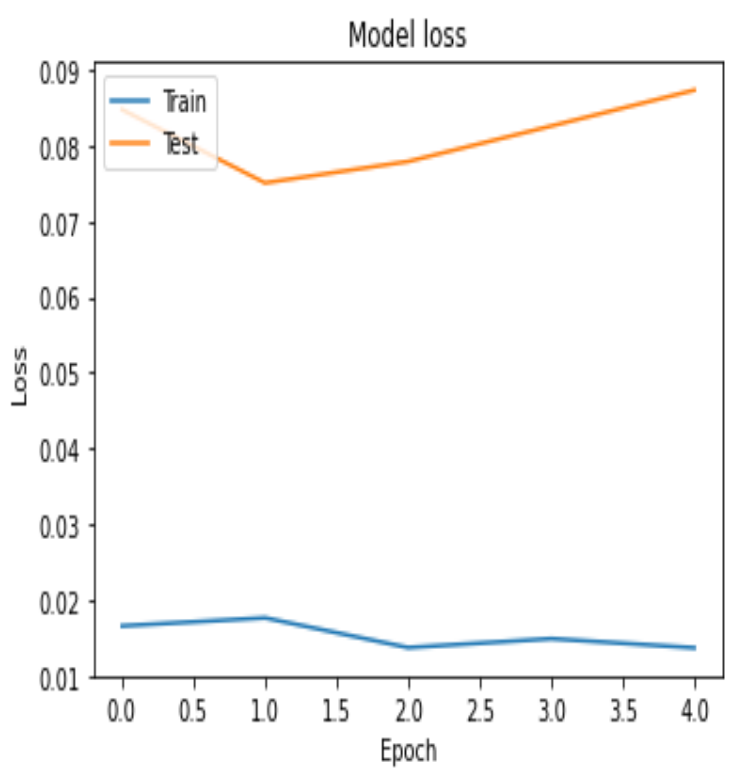

(b)

Figure 13. Predicted electrical motor power analysis. (a) Behaviour of the predicted electrical motor power; (b) Model loss with increase in epoch.

\subsection{Prediction of Range of PEV Using the Single Output Model}

In this case, the range datasets were fed into the system as the desired variable while other variable were the inputs. The system was trained and validated. The overall model loss for training was 0.0057 . The overall model loss for testing was 0.0053 . Table 10 shows the predicted and the desired variables. Figure 14a shows the overlapping profiles of the predicted and the desired variables. The degree of overlap is between $85-93 \%$. Figure $14 \mathrm{~b}$ indicates the model losses as epoch increases. The training error was almost constant for the first two epochs while the testing error was changing for the entire process. 
Table 10. Predicted and desired ranges.

\begin{tabular}{ccccccccccc}
\hline & ComE & CE & ReT & HE & CGEq & HGEq & ComGEq & Mot & Ra & Predicted Ra \\
\hline 0 & 23.6 & 23.8 & 12 & 23.2 & 2.7 & 2.6 & 2.6 & 270 & 426 & 394.53876 \\
1 & 17.8 & 16.2 & 5 & 19.7 & 1.8 & 2.2 & 2 & 125 & 183 & 176.96542 \\
2 & 20.7 & 18.7 & 3 & 23.1 & 2.1 & 2.6 & 2.3 & 60 & 92 & 89.903847 \\
3 & 15.8 & 14.5 & 5.8 & 17.4 & 1.6 & 1.9 & 1.8 & 100 & 274 & 263.87628 \\
4 & 18.9 & 18.2 & 10 & 19.8 & 2 & 2.2 & 2.1 & 358 & 488 & 463.54578 \\
5 & 19.3 & 16.8 & 5 & 22.4 & 1.9 & 2.5 & 2.2 & 81 & 179 & 172.93135 \\
6 & 18.6 & 17 & 6 & 20.7 & 1.9 & 2.3 & 2.1 & 80 & 172 & 164.85034 \\
7 & 17.4 & 16.8 & 5.3 & 18.6 & 1.9 & 2.1 & 2 & 100 & 201 & 197.63403 \\
8 & 17.4 & 16.3 & 10 & 18.7 & 1.8 & 2.1 & 2 & 358 & 507 & 524.77155 \\
9 & 21 & 19.9 & 8.8 & 22.4 & 2.2 & 2.5 & 2.4 & 198 & 370 & 399.02506 \\
\hline
\end{tabular}

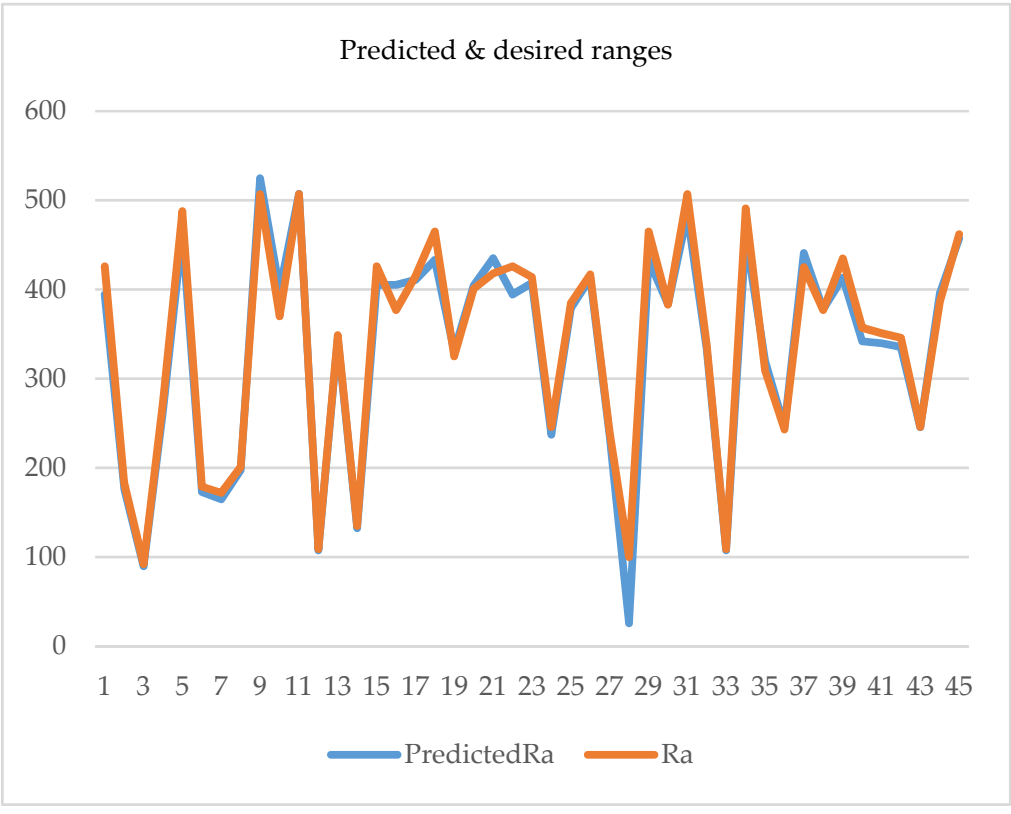

(a)

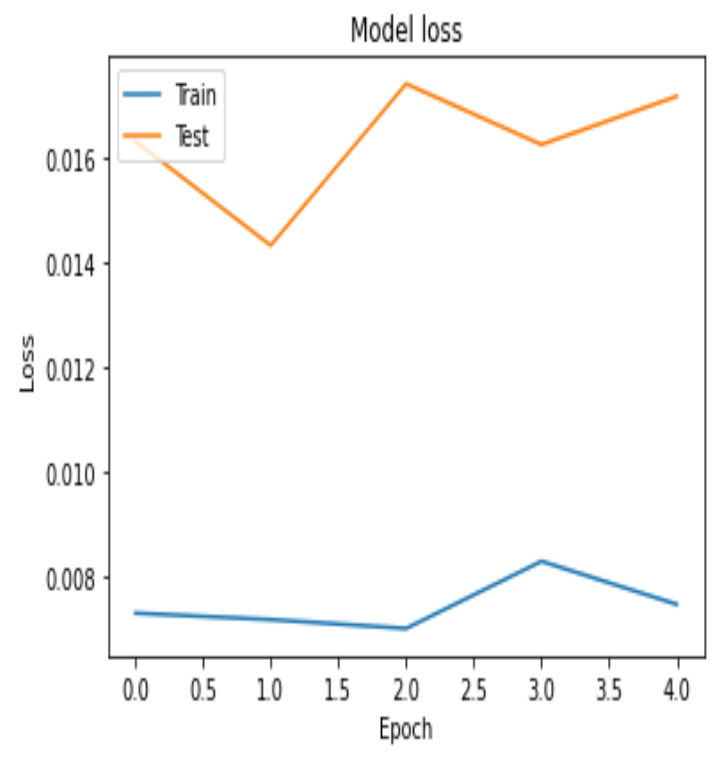

(b)

Figure 14. Predicted range analysis. (a) Behaviour of the predicted range; (b) Model loss with increase in epoch.

\section{Discussion}

\subsection{Predictability of the Proposed Model}

Considering the main target variable in Section 3.1 that was used for the model formation, the overall losses for the training and testing were $3.9132 \times 10^{-6}$ and $9.698 \times 10^{-7}$, respectively. The mean absolute error (MAE), mean square error (MSE) and root mean square error (RMSE) were $0.109189,0.218379$, and 0.46731 , respectively. The main target variable, the combined electrical charge consumption, had an extraordinary prediction result.

The same model was used to predict the other eight variables, yielding predicted outputs with negligible overall training, and testing errors as describe in Sections 3.2-3.9. This shows that the model can be adopted for simulation of any of the nine parameters both locally (in Canada) and internationally. Canada was used as a case study because of the high target set by the Canadian government to minimize greenhouse gas emissions. More so, another reason for this is because the research was being conducted in Canada. 


\subsection{Applicability of the Proposed Model to Variety of Designs}

This model is applicable to different categories of pure electric cars. The categories of the cars which the model is found applicable are two-seater, full-size, compact, subcompact, mid-size, standard SUV, and small station wagons. The model can be used to simulate any of the nine parameters for the following makes of vehicles: Mitsubishi, Nissan, Ford, Smart, Tesla, Chevrolet, BMW, Kia, Hyundai, Audi, Volkswagen, Porsche, and Volvo. It has not been tested with the datasets of commercial pure electric buses and trucks.

\subsection{Implications of the Developed Model and Technique}

\subsubsection{Formulation of Policy}

Base on the capability of model to predict city electric charge consumption, highway electrical charge consumption and combined electric charge consumption, the governmental regulation agencies can formulate standard figures for manufacturers and users of PEVs. For example, the government can establish the electric charge consumption limits for new categories of PEVs to be manufactured. The government or its agencies can establish the cost of recharging the batteries per kilowatt hour. The government can also generate taxes base on the consumption of electric charges per $\mathrm{km}$.

\subsubsection{Decision Making by the Manufacturers of PEVs}

Manufacturers of PEVs and batteries can use this model to make certain critical decisions at the point of design. For instance, the simulation of electrical motor power can help in deciding the selection of battery type, density, and capacity for a car to be designed. A manufacturer can determine the capacity of the battery to be used if the range is simulated. The higher the range, the higher the capacity of the battery to be used for the design of PEVs. There is a linear relationship between the range and battery density. Vehicle spare parts manufacturers can benefit maximally by employing the model. Battery manufacturers can use the model to predict car ranges for their batteries. This will help in battery capacity estimation. Car manufacturers can also use the model to predict the rate of consumption of electrical charges per $\mathrm{km}$.

\subsubsection{Impact of Government Policy}

Government policies can play vital roles in the demand and supply of PEVs. Enacting zero-emission policy can increase the demand of PEVs. The is because people, companies and government parastatals would be mandated to purchase PEVs. In addition, provision of subsidies or credits by the government to the buyers of PEVs can also increase the demand of PEVs. This is because when the selling price of PEVs is lowered, more people will be willing to buy them. The supply of PEVs can also be positively influenced if government provide financial aids to manufacturers of PEVs. Such supportive policies mentioned above would promote the usage of this model due to increase in demand and supply of PEVs.

\subsection{Comparativeness with the Past Studies}

Looking at the past works on predictions with ANN, most of these studies cannot predicted nine variables of PEVs with a single model. In addition, most of these works focused on energy management $[13,14,16,21-23,25]$ and batteries [18-20,28] predictions. This current study addresses the problem of PEVs with respect to manufacturing and increase in demand. The developed model can be used to simulate the nine designparameters accurately. This will have positive impact on the speed and accuracy of the future designs if the model is employed. Customers and manufacturers will also be able to make decisions on time and accurately. 


\subsection{Validation with the Redefined Parameters}

The model was used to predict variables using redefined datasets. The predictions with redefined datasets equally indicated high degree of accuracy. Table 11a-i and Figure 15a-i show the results of the prediction for all the nine variables. The last two columns of Table 11a-i show the desired and the predicted variables for each of the nine situations. Nine different situations were presented in Table 11a-i. The first is the prediction of city electric charge consumption in Table 11a. The absolute errors between the desired and predicted variables from this table were negligible. The mean absolute error for this case is 0.0592 . Table $11 \mathrm{~b}$ indicated the prediction result for the highway electric charge consumption. The absolute errors between the desired and predicted variables from this table were minimal. The mean absolute error for this case is 0.06426 . Table $11 \mathrm{c}$ indicated the prediction result of the electrical motor power. This table shows the absolute errors between the target and the desired variables. The mean absolute error for this case is 0.3191 . Table $11 \mathrm{~d}$ shows city gasoline litre consumption equivalent prediction result. The absolute errors between the target and predicted variables from this table were small. The mean absolute error for this case is 0.06405 . Table 11e shows highway gasoline litre consumption equivalent. The absolute errors between the desired and predicted variables from this table were also negligible. The mean absolute error for this case is 0.09516 . The combined gasoline litre consumption equivalent prediction is shown in Table 11f. The absolute errors between the desired and predicted variables from this table were small. The mean absolute error for this prediction is 0.1136 . The recharge time result is indicated in Table $11 \mathrm{~g}$. The absolute errors between the target and predicted variables from this table were small. The mean absolute error for this case is 0.2449 . Table $11 \mathrm{~h}$ shows the prediction result for range. The mean absolute error for the prediction is 0.20494 . Table $11 \mathrm{i}$ indicated the details of the combined electric charge consumption result. The absolute errors between the target and predicted variables from this table were small. The mean absolute error for this case is 0.0356 . All these prediction results for the redefined cases are of high degree of accuracy.

Table 11. Redefined parametric predictions.

(a) City electric charge consumption

\begin{tabular}{|c|c|c|c|c|c|c|c|c|c|c|}
\hline & HE & ComE & Mot & $\mathbf{R a}$ & CGEq & HGEq & ComGEq & $\operatorname{ReT}$ & $\mathrm{CE}$ & Predicted CE \\
\hline 0 & 23.2 & 23.6 & 270 & 426 & 2.7 & 2.6 & 2.6 & 12 & 46.6 & 47.066566 \\
\hline 1 & 19.7 & 17.8 & 125 & 183 & 1.8 & 2.2 & 2 & 5 & 31.4 & 31.082979 \\
\hline 2 & 23.1 & 20.7 & 60 & 92 & 2.1 & 2.6 & 2.3 & 3 & 36.4 & 36.024353 \\
\hline 3 & 17.4 & 15.8 & 100 & 274 & 1.6 & 1.9 & 1.8 & 5.8 & 28 & 27.530424 \\
\hline 4 & 19.8 & 18.9 & 358 & 488 & 2 & 2.2 & 2.1 & 10 & 35.4 & 34.879467 \\
\hline 5 & 22.4 & 19.3 & 81 & 179 & 1.9 & 2.5 & 2.2 & 5 & 32.6 & 32.706402 \\
\hline 6 & 20.7 & 18.6 & 80 & 172 & 1.9 & 2.3 & 2 & 6 & 33 & 32.533249 \\
\hline 7 & 18.6 & 17.4 & 100 & 201 & 1.9 & 2.1 & 2 & 5.3 & 32.6 & 31.684757 \\
\hline 8 & 22.3 & 17.4 & 358 & 507 & 1.9 & 2.1 & 2.4 & 10 & 31.6 & 31.951500 \\
\hline 9 & 20 & 21 & 198 & 370 & 1.8 & 2.5 & 2.4 & 8.8 & 38.8 & 38.254368 \\
\hline \multicolumn{11}{|c|}{ (b) Highway electric charge consumption } \\
\hline & $\mathrm{CE}$ & ComE & Mot & $\mathbf{R a}$ & CGEq & HGEq & ComGEq & $\operatorname{ReT}$ & HE & Predicted HE \\
\hline 0 & 23.8 & 23.6 & 270 & 426 & 2.7 & 2.6 & 2.6 & 12 & 23.2 & 23.21851 \\
\hline 1 & 16.2 & 17.8 & 125 & 183 & 1.8 & 2.2 & 2 & 5 & 19.7 & 19.406933 \\
\hline 2 & 18.7 & 20.7 & 60 & 92 & 2.1 & 2.6 & 2.3 & 3 & 23.1 & 23.057276 \\
\hline 3 & 14.5 & 15.8 & 100 & 274 & 1.6 & 1.9 & 1.8 & 5.8 & 17.4 & 17.038816 \\
\hline 4 & 18.2 & 18.9 & 358 & 488 & 2 & 2.2 & 2.1 & 10 & 19.8 & 19.505558 \\
\hline 5 & 16.8 & 19.3 & 81 & 179 & 1.9 & 2.5 & 2.2 & 5 & 22.4 & 22.123541 \\
\hline 6 & 17 & 18.6 & 80 & 172 & 1.9 & 2.3 & 2 & 6 & 20.7 & 20.51317 \\
\hline 7 & 16.8 & 17.4 & 100 & 201 & 1.9 & 2.1 & 2 & 5.3 & 18.6 & 18.596674 \\
\hline 8 & 16.3 & 17.4 & 358 & 507 & 1.9 & 2.1 & 2.4 & 10 & 18.7 & 18.57807 \\
\hline 9 & 19.9 & 21 & 198 & 370 & 1.8 & 2.5 & 2.4 & 8.8 & 22.4 & 22.484404 \\
\hline
\end{tabular}


Table 11. Cont.

(c) Electric motor power

\begin{tabular}{ccccccccccc}
\hline & HE & ReT & CGEq & HGEq & ComGEq & Ra & CE & ComE & Mot & Predicted Mot \\
\hline 0 & 23.2 & 12 & 2.7 & 2.6 & 2.6 & 426 & 23.8 & 23.6 & 140 & 200.348709 \\
1 & 19.7 & 5 & 1.8 & 2.2 & 2 & 183 & 16.2 & 17.8 & 67.5 & 61.672386 \\
2 & 23.1 & 3 & 2.1 & 2.6 & 2.3 & 92 & 18.7 & 20.7 & 35 & 31.928759 \\
3 & 17.4 & 5.8 & 1.6 & 1.9 & 1.8 & 274 & 14.5 & 15.8 & 55 & 63.545692 \\
4 & 19.8 & 10 & 2 & 2.2 & 2.1 & 488 & 18.2 & 18.9 & 184 & 189.540115 \\
5 & 22.4 & 5 & 1.9 & 2.5 & 2.2 & 179 & 16.8 & 19.3 & 45.5 & 46.0271 \\
6 & 20.7 & 6 & 1.9 & 2.3 & 2 & 172 & 17 & 18.6 & 45 & 44.363953 \\
7 & 18.6 & 5.3 & 1.9 & 2.1 & 2 & 201 & 16.8 & 17.4 & 55 & 56.778175 \\
8 & 18.7 & 10 & 1.8 & 2.1 & 2.4 & 507 & 16.3 & 17.4 & 184 & 156.964767 \\
9 & 22.4 & 8.8 & 2.2 & 2.5 & 2.4 & 370 & 19.9 & 21 & 104 & 124.466232 \\
\hline
\end{tabular}

(d) City gasoline litre consumption equivalent

\begin{tabular}{ccccccccccc}
\hline & HE & HGEq & ReT & CE & ComE & Mot & Ra & ComGEq & CGEq & Predicted CGEq \\
\hline 0 & 23.2 & 2.6 & 12 & 23.8 & 23.6 & 270 & 426 & 2.6 & 15.29 & 15.02135181 \\
1 & 19.7 & 2.2 & 5 & 16.2 & 17.8 & 125 & 183 & 2 & 11.24 & 11.35358334 \\
2 & 23.1 & 2.6 & 3 & 18.7 & 20.7 & 60 & 92 & 2.3 & 12.41 & 12.51209927 \\
3 & 17.4 & 1.9 & 5.8 & 14.5 & 15.8 & 100 & 274 & 1.8 & 10.56 & 10.59348011 \\
4 & 19.8 & 2.2 & 10 & 18.2 & 18.9 & 358 & 488 & 2.1 & 12 & 12.15197659 \\
5 & 22.4 & 2.5 & 5 & 16.8 & 19.3 & 81 & 179 & 2.2 & 11.61 & 11.71028996 \\
6 & 20.7 & 2.3 & 6 & 17 & 18.6 & 80 & 172 & 2 & 11.61 & 11.59972286 \\
7 & 18.6 & 2.1 & 5.3 & 16.8 & 17.4 & 100 & 201 & 2 & 11.61 & 11.36447811 \\
8 & 18.7 & 2.1 & 10 & 16.3 & 17.4 & 358 & 507 & 2.4 & 11.24 & 11.49509335 \\
9 & 22.4 & 2.5 & 8.8 & 19.9 & 21 & 198 & 370 & 2.4 & 12.84 & 12.95093346 \\
\hline
\end{tabular}

(e) Highway gasoline litre consumption equivalent

\begin{tabular}{ccccccccccc}
\hline & HE & ReT & CE & ComE & Mot & Ra & ComGEq & CGEq & HGEq & Predicted HGEq \\
\hline 0 & 23.2 & 12 & 23.8 & 23.6 & 270 & 426 & 2.6 & 2.7 & 17.576 & 17.74665260 \\
1 & 19.7 & 5 & 16.2 & 17.8 & 125 & 183 & 2 & 1.8 & 10.648 & 10.65536785 \\
2 & 23.1 & 3 & 18.7 & 20.7 & 60 & 92 & 2.3 & 2.1 & 17.576 & 18.37563324 \\
3 & 17.4 & 5.8 & 14.5 & 15.8 & 100 & 274 & 1.8 & 1.6 & 6.859 & 7.435733795 \\
4 & 19.8 & 10 & 18.2 & 18.9 & 358 & 488 & 2.1 & 2 & 10.648 & 10.56519318 \\
5 & 22.4 & 5 & 16.8 & 19.3 & 81 & 179 & 2.2 & 1.9 & 15.625 & 15.85181236 \\
6 & 20.7 & 6 & 17 & 18.6 & 80 & 172 & 2 & 1.9 & 12.167 & 12.52617073 \\
7 & 18.6 & 5.3 & 16.8 & 17.4 & 100 & 201 & 2 & 1.9 & 9.261 & 9.117634773 \\
8 & 18.7 & 10 & 16.3 & 17.4 & 358 & 507 & 2.4 & 1.8 & 9.261 & 9.006069183 \\
9 & 22.4 & 8.8 & 19.9 & 21 & 198 & 370 & 2.4 & 2.2 & 15.625 & 15.56515598 \\
\hline
\end{tabular}

(f) Combined gasoline litre consumption equivalent

\begin{tabular}{ccccccccccc}
\hline & HE & ReT & CE & ComE & Mot & Ra & CGEq & HGEq & ComGEq & Predicted ComGEq \\
\hline 0 & 23.2 & 12 & 23.8 & 23.6 & 270 & 426 & 2.7 & 2.6 & 15.52 & 16.26508331 \\
1 & 19.7 & 5 & 16.2 & 17.8 & 125 & 183 & 1.8 & 2.2 & 10 & 9.779075623 \\
2 & 23.1 & 3 & 18.7 & 20.7 & 60 & 92 & 2.1 & 2.6 & 12.58 & 12.86681652 \\
3 & 17.4 & 5.8 & 14.5 & 15.8 & 100 & 274 & 1.6 & 1.9 & 8.48 & 8.135276794 \\
4 & 19.8 & 10 & 18.2 & 18.9 & 358 & 488 & 2 & 2.2 & 10.82 & 11.03121662 \\
5 & 22.4 & 5 & 16.8 & 19.3 & 81 & 179 & 1.9 & 2.5 & 11.68 & 11.22401524 \\
6 & 20.7 & 6 & 17 & 18.6 & 80 & 172 & 1.9 & 2.3 & 10.82 & 10.58418751 \\
7 & 18.6 & 5.3 & 16.8 & 17.4 & 100 & 201 & 1.9 & 2.1 & 10 & 9.605104446 \\
8 & 18.7 & 10 & 16.3 & 17.4 & 358 & 507 & 1.8 & 2.1 & 10 & 9.768924713 \\
9 & 22.4 & 8.8 & 19.9 & 21 & 198 & 370 & 2.2 & 2.5 & 13.52 & 12.71550655 \\
\hline
\end{tabular}


Table 11. Cont.

(g) Recharge time

\begin{tabular}{ccccccccccc}
\hline & HE & ComGEq & CE & ComE & Mot & Ra & CGEq & HGEq & ReT & Predicted ReT \\
\hline 0 & 23.2 & 2.6 & 23.8 & 23.6 & 270 & 426 & 2.7 & 2.6 & 19 & 18.99589539 \\
1 & 19.7 & 2 & 16.2 & 17.8 & 125 & 183 & 1.8 & 2.2 & 5 & 5.247741699 \\
2 & 23.1 & 2.3 & 18.7 & 20.7 & 60 & 92 & 2.1 & 2.6 & 1 & 1.816795230 \\
3 & 17.4 & 1.8 & 14.5 & 15.8 & 100 & 274 & 1.6 & 1.9 & 6.6 & 6.215961456 \\
4 & 19.8 & 2.1 & 18.2 & 18.9 & 358 & 488 & 2 & 2.2 & 15 & 15.38726711 \\
5 & 22.4 & 2.2 & 16.8 & 19.3 & 81 & 179 & 1.9 & 2.5 & 5 & 5.549118042 \\
6 & 20.7 & 2 & 17 & 18.6 & 80 & 172 & 1.9 & 2.3 & 7 & 7.311823845 \\
7 & 18.6 & 2 & 16.8 & 17.4 & 100 & 201 & 1.9 & 2.1 & 5.6 & 5.555485725 \\
8 & 18.7 & 2.4 & 16.3 & 17.4 & 358 & 507 & 1.8 & 2.1 & 15 & 14.07801914 \\
9 & 22.4 & 2.4 & 19.9 & 21 & 198 & 370 & 2.2 & 2.5 & 12.6 & 9.335071564 \\
\hline
\end{tabular}

(h) Range

\begin{tabular}{ccccccccccc}
\hline & HE & ComGEq & CE & ComE & Mot & ReT & CGEq & HGEq & Ra & Predicted Ra \\
\hline 0 & 23.2 & 2.6 & 23.8 & 23.6 & 270 & 12 & 2.7 & 2.6 & 260.6 & 247.2724609 \\
1 & 19.7 & 2 & 16.2 & 17.8 & 125 & 5 & 1.8 & 2.2 & 114.8 & 105.6697540 \\
2 & 23.1 & 2.3 & 18.7 & 20.7 & 60 & 3 & 2.1 & 2.6 & 60.2 & 66.36408997 \\
3 & 17.4 & 1.8 & 14.5 & 15.8 & 100 & 5.8 & 1.6 & 1.9 & 169.4 & 155.8714142 \\
4 & 19.8 & 2.1 & 18.2 & 18.9 & 358 & 10 & 2 & 2.2 & 297.8 & 299.7419739 \\
5 & 22.4 & 2.2 & 16.8 & 19.3 & 81 & 5 & 1.9 & 2.5 & 112.4 & 108.7931900 \\
6 & 20.7 & 2 & 17 & 18.6 & 80 & 6 & 1.9 & 2.3 & 108.2 & 91.59775543 \\
7 & 18.6 & 2 & 16.8 & 17.4 & 100 & 5.3 & 1.9 & 2.1 & 125.6 & 120.0908890 \\
8 & 18.7 & 2.4 & 16.3 & 17.4 & 358 & 10 & 1.8 & 2.1 & 309.2 & 336.3500977 \\
9 & 22.4 & 2.4 & 19.9 & 21 & 198 & 8.8 & 2.2 & 2.5 & 227 & 246.8524475 \\
\hline
\end{tabular}

(i) Combined electric charge consumption

\begin{tabular}{|c|c|c|c|c|c|c|c|c|c|c|}
\hline & HE & $\operatorname{ReT}$ & CGEq & HGEq & ComGEq & Mot & $\mathbf{R a}$ & CE & ComE & Predicted ComE \\
\hline 0 & 23.2 & 12 & 2.7 & 2.6 & 2.6 & 270 & 260.6 & 23.8 & 60.8 & 60.34340286 \\
\hline 1 & 19.7 & 5 & 1.8 & 2.2 & 2 & 125 & 114.8 & 16.2 & 43.4 & 43.52837372 \\
\hline 2 & 23.1 & 3 & 2.1 & 2.6 & 2.3 & 60 & 60.2 & 18.7 & 52.1 & 51.92724609 \\
\hline 3 & 17.4 & 5.8 & 1.6 & 1.9 & 1.8 & 100 & 169.4 & 14.5 & 37.4 & 37.66779327 \\
\hline 4 & 19.8 & 10 & 2 & 2.2 & 2.1 & 358 & 297.8 & 18.2 & 46.7 & 46.68238449 \\
\hline 5 & 22.4 & 5 & 1.9 & 2.5 & 2.2 & 81 & 112.4 & 16.8 & 47.9 & 47.92834473 \\
\hline 6 & 20.7 & 6 & 1.9 & 2.3 & 2 & 80 & 108.2 & 17 & 45.8 & 45.81203461 \\
\hline 7 & 18.6 & 5.3 & 1.9 & 2.1 & 2 & 100 & 125.6 & 16.8 & 42.2 & 42.57527161 \\
\hline 8 & 18.7 & 10 & 1.8 & 2.1 & 2.4 & 358 & 309.2 & 16.3 & 42.2 & 42.24062347 \\
\hline 9 & 22.4 & 8.8 & 2.2 & 2.5 & 2.4 & 198 & 227 & 19.9 & 53 & 52.74155426 \\
\hline
\end{tabular}

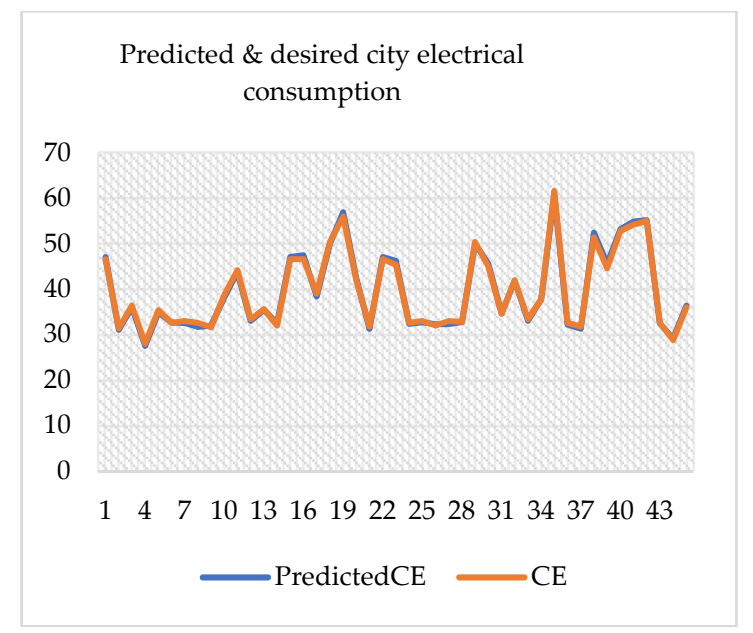

(a)

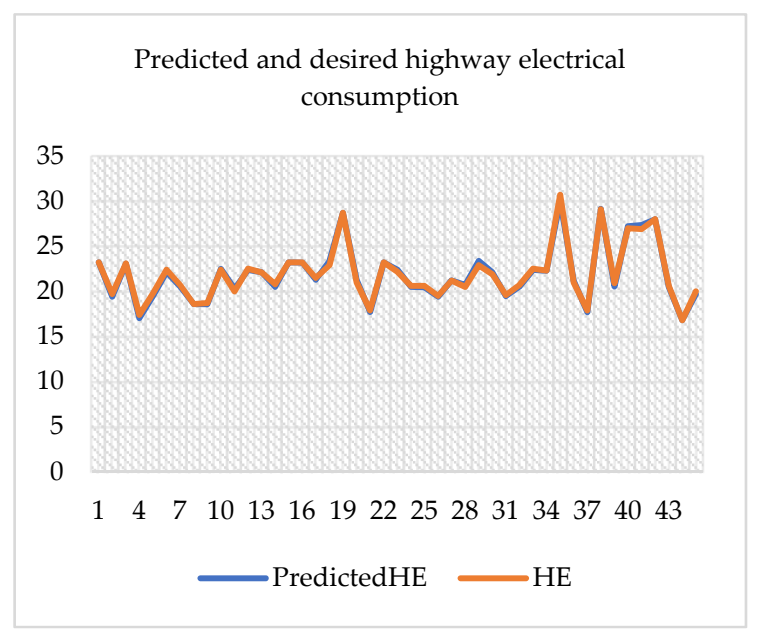

(b)

Figure 15. Cont. 


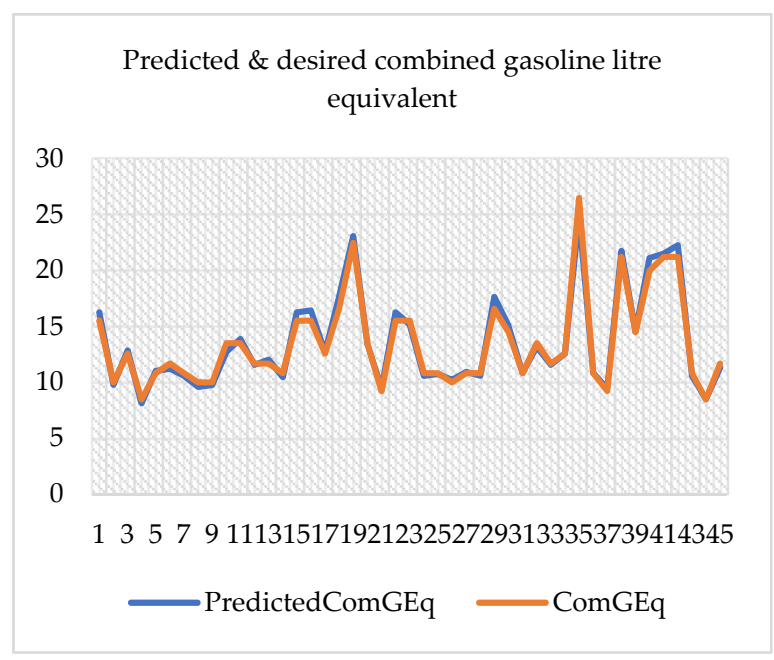

(c)

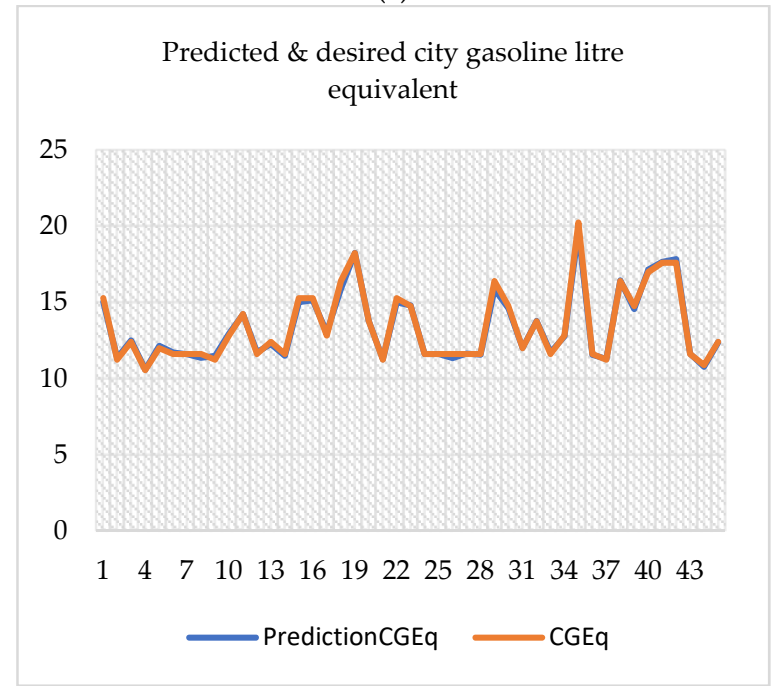

(e)

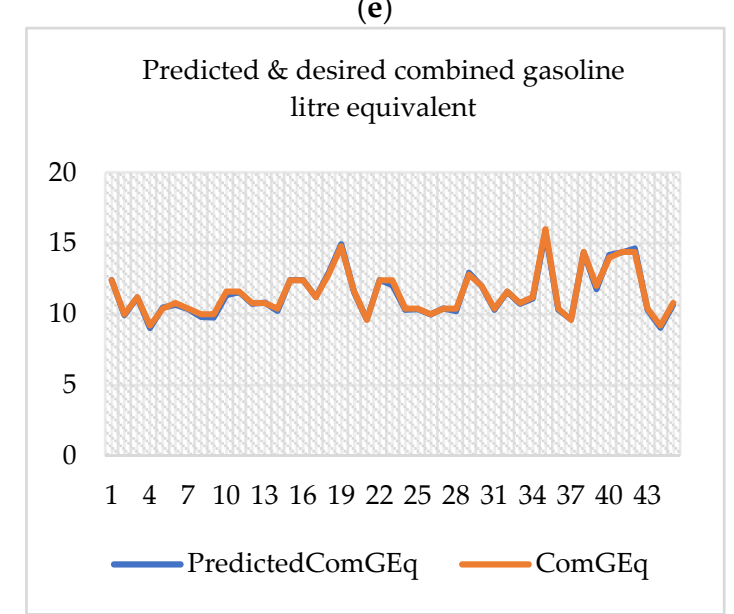

(g)

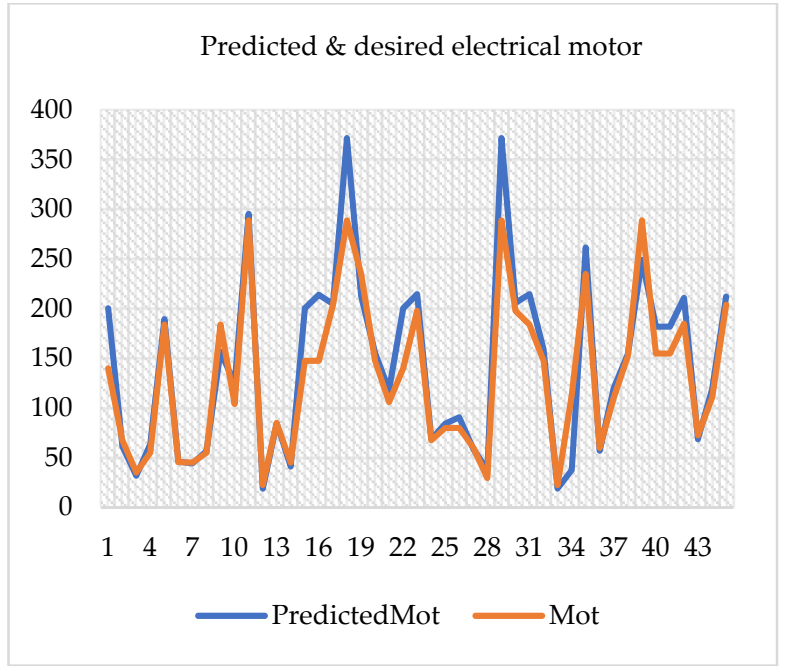

(d)

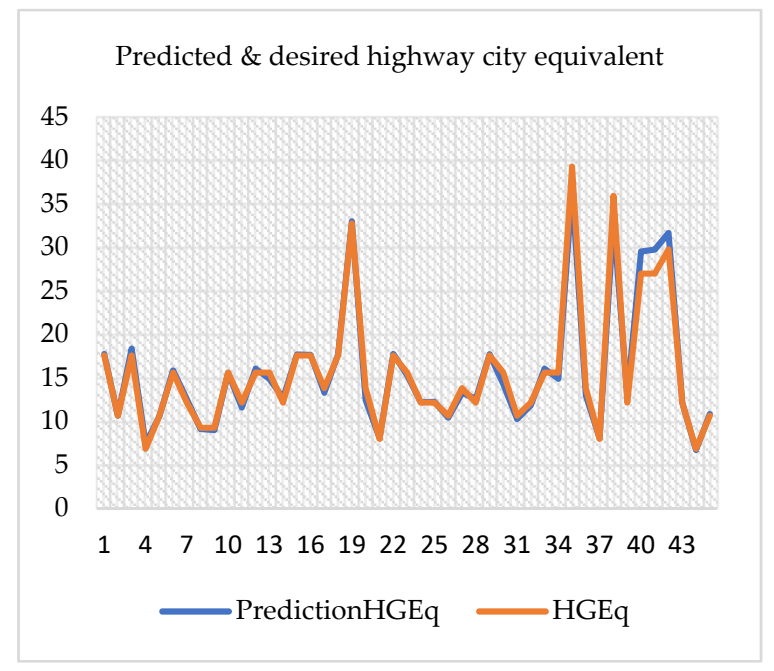

(f)

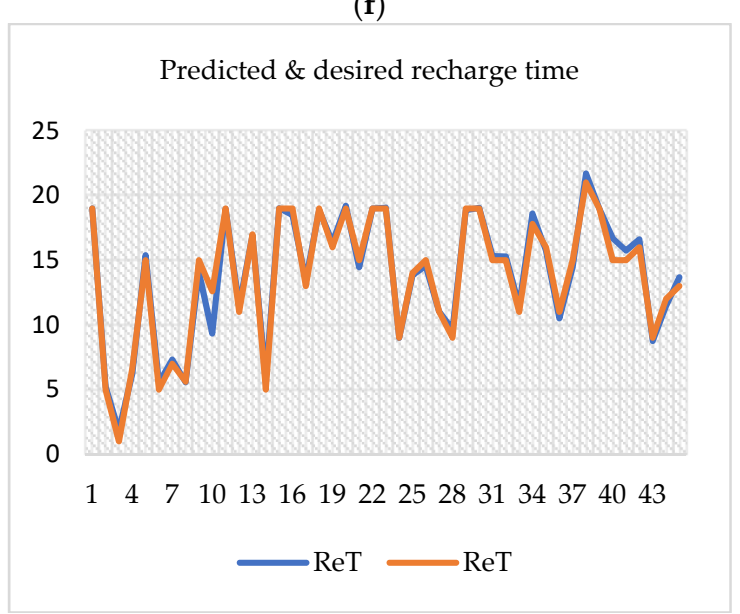

(h)

Figure 15. Cont. 


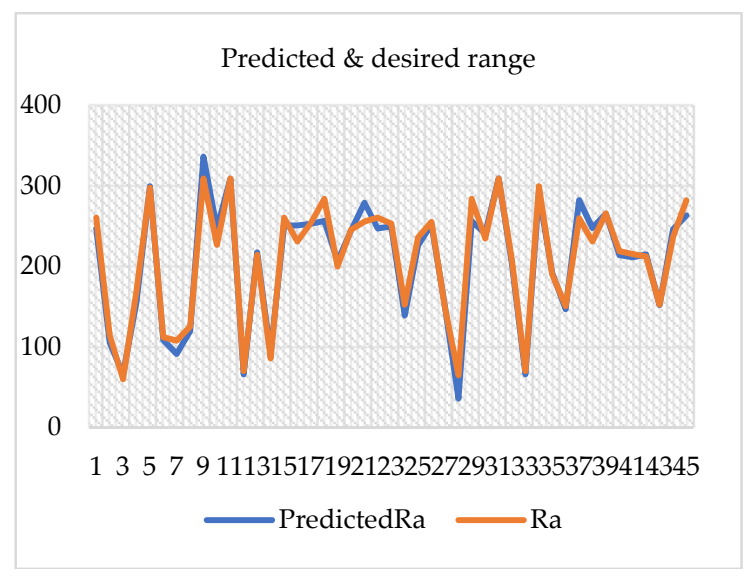

(i)

Figure 15. Redefined parametric predictions performance measurement. (a) City electric charge consumption; (b) Highway electric charge consumption; (c) Combined electric charge consumption; (d) Electric motor power; (e) City gasoline litre consumption equivalent; (f) Highway gasoline litre consumption equivalent; (g) Combined gasoline litre consumption equivalent; (h) Recharge Time; (i) Range.

Figure 15a-i are comparative analysis between the predicted and the desired variables. For majority of the parameters, the desired variable overlapped with the predicted variable for up to $75-95 \%$. This signifies high degree of accuracy for the predictions.

Table 12 and Figure 16 show the details of the mean absolute error (MAE), mean square error (MSE), room mean square errors (RMSE), for the redefined parameters in Section 4.5. From Figure 16 and Table 12, it can be shown that the electrical motor power (Mot) is the parameter with the highest MAE, MSE and RMSE. The parameter with the lowest MAE, MSE and RMSE is the combined electrical charge consumption (ComE). If 0.5 is assumed to be the minimum allowable mean value for the error functions, then the losses are said to be minimal.

Table 12. Model evaluation using redefined parameters.

\begin{tabular}{cccc}
\hline Parameter & MAE & MSE & RMSE \\
\hline CGEq & 0.06405 & 0.0082 & 0.0910 \\
HGEq & 0.09516 & 0.02277 & 0.15089 \\
ComGEq & 0.1136 & 0.02266 & 0.1505 \\
ReT & 0.2449 & 0.1192 & 0.3454 \\
CE & 0.0592 & 0.004590 & 0.06774 \\
HE & 0.06426 & 0.006881 & 0.08295 \\
ComE & 0.03567 & 0.003285 & 0.05732 \\
Ra & 0.20494 & 0.05750 & 0.2398 \\
Mot & 0.3191 & 0.2073 & 0.4553 \\
\hline
\end{tabular}

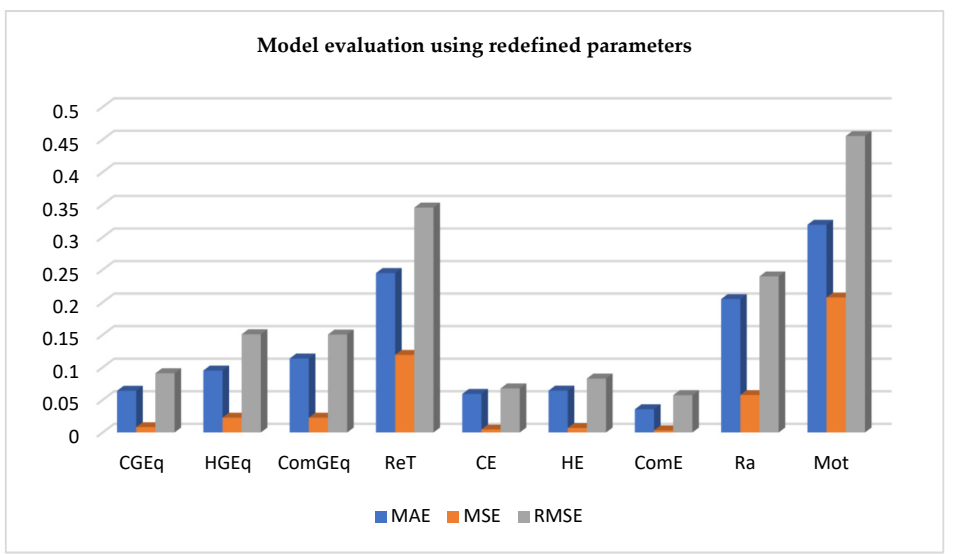

Figure 16. Model evaluation with the redefined parameters. 


\subsection{Evaluation of the Model with Error Functions}

Table 13 shows the catalogue of training and testing errors for the prediction of all the nine parameters in Section 3. Figure 17 shows the training and the testing error profiles using the values in Table 13. Figures 17 and 18 also show the relationship between the training and the testing errors for all the variables. From Figure 18, the electrical motor power has the highest training error while the combined electrical charge consumption has the lowest value. The recharge time for the battery has the highest error value for testing while the combined electrical consumption has the lowest value.

Table 13. Training and testing errors.

\begin{tabular}{ccc}
\hline Parameters & Training Errors & Testing Errors \\
\hline Mot & 0.0181 & 0.0053 \\
CE & 0.0071 & 0.0021 \\
HE & 0.0013 & 0.0013 \\
ComE & $3.9132 \times 10^{-6}$ & $9.698 \times 10^{-7}$ \\
HGEq & 0.0039 & 0.0045 \\
CGEq & 0.0039 & 0.0045 \\
ComGEq & 0.0017 & 0.0016 \\
Ra & 0.0057 & 0.0053 \\
ReT & 0.0011 & 0.0058932 \\
\hline
\end{tabular}

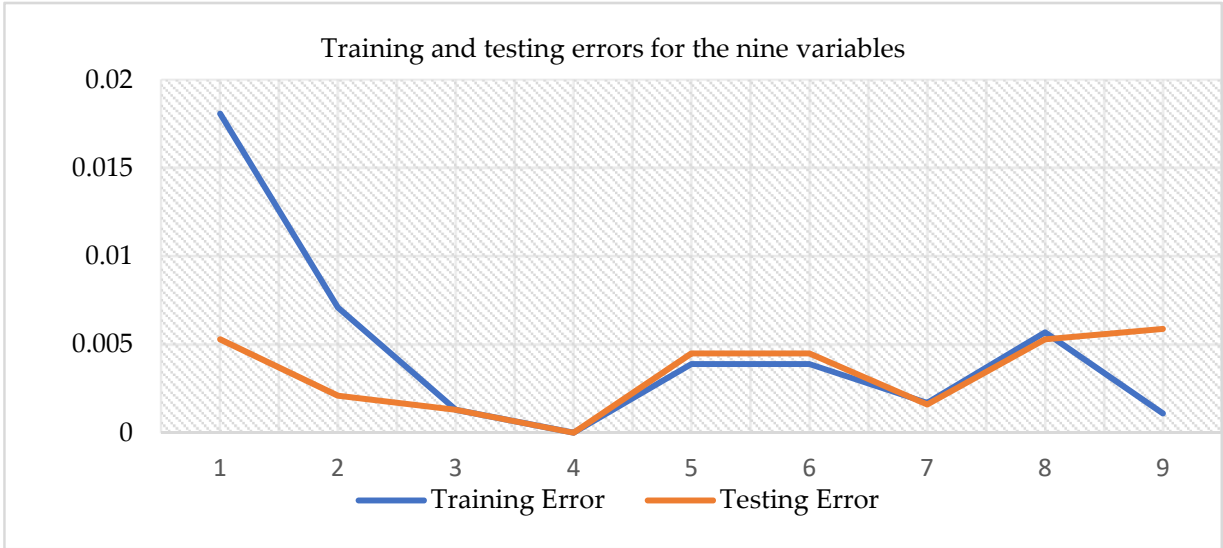

Figure 17. Training and testing errors for the nine variables.

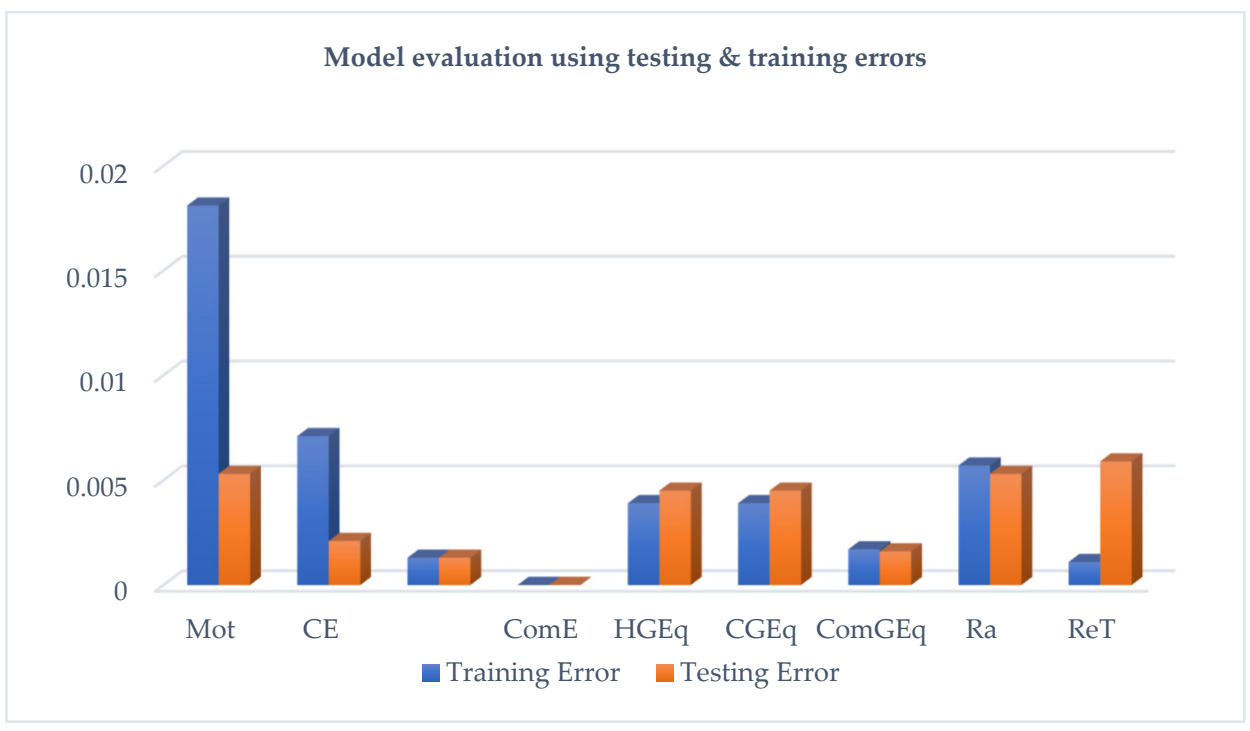

Figure 18. Model evaluation. 


\section{Conclusions and Future Works}

\subsection{Conclusions}

This study presented a unique technique for predicting the parameters of PEVs for manufacturing purposes. A Multilayer Artificial Neural Network model was developed by using a direct model approach of ANNs. Nine variables were predicted using datasets from the manufacturers of PEVs. The proposed model was also evaluated using redefined datasets. For the main variable, the combined electrical charge consumption, the mean absolute error, mean square error and root mean square errors of the model were 0.109 , 0.218 and 0.467 , respectively. For the model formation, using the combined electrical charge consumption as the target variable, the overall losses for the training and testing were $3.9132 \times 10^{-6}$ and $9.698 \times 10^{-7}$, respectively. The predicted results were of high degree of accuracy. The following are the contributions of this study:

- A proposed predicting model was developed for pure electric vehicles parametric simulations. The model will help manufacturers, spare part producers and policy makers, in making decision more accurately and promptly.

- The built model can predict all the selected nine parameters of the pure electric vehicles. The nine variables were predicted using the model without changing any parameter of the model. This attested to the high efficiency and high accuracy of the model.

- The proposed model can be used industrially to predict future electric vehicle variables. Hence, it can serve as predicting machine for manufacturing of PEVs.

\subsection{Future Works}

This study can be improved upon as future research works. The work is limited to cars of different categories. The future work may include datasets of pure electric trucks and buses. Energy management datasets such as speed, electric current, electric voltage can be included in the future research. Future research may develop a single model for PEVs, PHEVs and HEVs.

Funding: Saskatchewan Innovation and Excellence Funding, ID:36002.

Institutional Review Board Statement: Not applicable.

Informed Consent Statement: Not applicable.

Data Availability Statement: The data is available on the website of Ministry of Transportation Canada. Fuel consumption ratings search tool. Available online: https:/ /www.nrcan-rncan.gc.ca (accessed on 14 March 2021).

Conflicts of Interest: The authors declare no conflict of interest.

\section{References}

1. Exclusive Biden Electric Vehicle Includes Battery Recycling Push. Available online: https//www.reuters.com/business/autostransportation/exclusive-bidens-electric-vehicle-plan-includes-battery-recycling-push-2021-06-04 (accessed on 4 June 2021).

2. Natural Resources Canada. Fuel Consumption Ratings Search Tool. Available online: https//www.nrcan-rncan.gc.ca (accessed on 14 March 2021).

3. Şafak, B.I.; Ali, T. Introduction to Plug-in Electric Vehicles, Plug-In Electric Vehicle Grid Integration; Artech House: Norwood, MA, USA, 2017.

4. Types of Electric Vehicles: A Short Guide to Electric Cars-Ezoomed. Available online: www.ezoomed.com/blog/buy-new/ types-electric-vehicles (accessed on 28 October 2021).

5. An Absolute Triumph of Efficiency, Lucid Air Achieves 520 miles of Range. Available online: https://www.lucidmotors.com/ stories/lucid-air-achieves-520-miles-of-range (accessed on 16 September 2021).

6. Yao, L.; Xiao, Y.; Gong, X.; Hou, J.; Chen, X. A Novel Intelligent Method for Fault Diagnosis of Electric Vehicle Battery System Based on Wavelet Neural Network. J. Power Sources 2020, 453, 227870. [CrossRef]

7. Chaoui, H.; Ibe-Ekeocha, C.C. State of Charge and State of Health Estimation for Lithium Batteries Using Recurrent Neural Networks. IEEE Trans. Veh. Technol. 2017, 66, 8773-8783. [CrossRef]

8. Xu, Q.; Mao, Y.; Zhao, M.; Cui, S. A Hybrid Electric Vehicle Dynamic Optimization Energy Management Strategy Based on a Compound-Structured Permanent-Magnet Motor. Energies 2018, 11, 2212. [CrossRef] 
9. Csaba, T.; John, J.C.; Ronald, P.J.; Nigel, N.C. Further Validation of Artificial Neural Network-Based Emissions Simulation Models for Conventional and Hybrid Electric Vehicles. J. Air Waste Manag. Assoc. 2006, 56, 898-910. [CrossRef]

10. Jiménez-Bermejo, D.; Fraile-Ardanuy, J.; Castaño-Solis, S.; Merino, J.; Álvaro-Hermana, R. Using Dynamic Neural Networks for Battery State of Charge Estimation in Electric Vehicles. Procedia Comput. Sci. 2018, 130, 533-540. [CrossRef]

11. Kumari, N.; Bhargava, V. Artificial Neural Network-Based Battery Energy Storage System for Electrical Vehicle; Springer Nature Singapore Pte Ltd.: Gateway East, Singapore, 2020.

12. Zainuri, A.; Wibawa, U.; Rusli, M.; Hasanah, R.N.; Harahap, R.A. VRLA battery state of health estimation based on charging time. Telkomnika Telecommun. Comput. Electron. Control. 2019, 17, 1577-1583. [CrossRef]

13. Chandran, V.; Patil, C.; Karthick, A.; Ganeshaperumal, D.; Rahim, R.; Ghosh, A. State of Charge Estimation of Lithium-Ion Battery for Electric Vehicles Using Machine Learning Algorithms. World Electr. Veh. J. 2021, 12, 38. [CrossRef]

14. Wang, Y.; Wu, Z.; Xia, A.; Guo, C.; Chen, Y.; Yang, Y.; Tang, Z. Energy management strategy for HEV based on KFCM and neural network. Concurr. Comput. Pract. Exp. 2019, 31, e4838. [CrossRef]

15. Shi, L.; Zheng, M.; Li, F. The energy management strategy for parallel hybrid electric vehicles based on MNN. Multimed. Tools Appl. 2020, 79, 5321-5333. [CrossRef]

16. Lin, X.; Wang, Z.; Wu, J. Energy management strategy based on velocity prediction using back propagation neural network for a plug-in fuel cell electric vehicle. Int. J. Energy Res. 2021, 45, 2629-2643. [CrossRef]

17. Sun, C.; Sun, F.; He, H. Investigating adaptive-ECMS with velocity forecast ability for hybrid electric vehicles. Appl. Energy 2017, 185, 1644-1653. [CrossRef]

18. Wang, Q.N.; Zeng, X.H.; Wang, P.Y.; Wang, J.N. Driving cycle recognition neural network algorithm based on the sliding time window for hybrid electric vehicles. Int. J. Automot. Technol. 2015, 16, 685-695. [CrossRef]

19. Wang, M.; Yu, H.; Dong, G.; Huang, M. Dual-Mode Adaptive Cruise Control Strategy Based on Model Predictive Control and Neural Network for Pure Electric Vehicles. In Proceedings of the 5th International Conference on Transportation Information and Safety, Liverpool, UK, 14-17 July 2019.

20. Zhu, J.; Yang, Z.; Mourshed, M.; Guo, Y.; Zhou, Y.; Chang, Y.; Wei, Y.; Feng, S. Electric Vehicle Charging Load Forecasting: A Comparative Study of Deep Learning Approaches. Energies 2019, 12, 2692. [CrossRef]

21. Xu, Y.; Zhang, W.; Bao, H.; Zhang, S.; Xiang, Y. A SEM-Neural Network Approach to Predict Customers' Intention to Purchase Battery Electric Vehicles in China's Zhejiang Province. Sustainability 2019, 11, 3164. [CrossRef]

22. Huang, H.B.; Wu, J.H.; Huang, X.R.; Yang, M.L.; Ding, W.P. The development of a deep neural network and its application to evaluating the interior sound quality of pure electric vehicles. Mech. Syst. Signal Process. 2019, 120, 98-116. [CrossRef]

23. Qian, K.; Hou, Z.; Sun, D. Sound Quality Estimation of Electric Vehicles Based on GA-BP Artificial Neural Networks. Appl. Sci. 2020, 10, 5567. [CrossRef]

24. Mayorga, R.M. Advanced Neural Network Lecture Note; University of Regina: Regina, SK, Canada, 2020.

25. Loy, J. Neural Network Projects with Python; Packt Publishing Ltd.: Birmingham, UK, 2019.

26. Kang, S.Y. An Investigation of the Use of Feedforward Neural Networks for Forecasting. Ph.D. Thesis, Kent State University, Kent, OH, USA, 1991.

27. Jang, J.S.R. Neuro-Fuzzy and Soft Computing: Adaptive Network; Prentice-Hall Inc.: Hoboken, NJ, USA, 1997; Chapter 8; pp. $226-233$.

28. James, L.; Lowry, J. Electric Vehicle Technology Explained; John Wiley \& Sons: Hoboken, NJ, USA, 2012.

29. All-Electric Vehicle. Available online: https:/ / Afdc.energy.gov (accessed on 5 September 2021).

30. Chris, M.; Abul Masur, M. Hybrid Electric Vehicles: Principles \& Application with Practical Perspectives; John Wiley \& Sons Ltd.: Hoboken, NJ, USA, 2018.

31. United States Environmental Protection Agency. Final Rule for Fuel Economy Labeling of Motor Vehicles: Revisions to Improve Calculation of Fuel Economy Estimates, 40 CFR Parts 86 and 600. 27 December 2006. Available online: http:/ / www.epa.gov/ fedrgstr/EPA-AIR/2006/December/Day-27/a9749.pdf (accessed on 14 March 2021). 